\title{
Smart exploitation of pseudorange and pseudorange-rate error characterization to improve the PVT solution
}

\author{
Eustachio Roberto Matera, Axel Garcia-Pena, Carl Milner, Bertrand Ekambi \\ Ecole Nationale de l'Aviation Civile (ENAC), ABBIA GNSS Technologies, Toulouse, France
}

\section{BIOGRAPHIES}

Eustachio Roberto Matera is currently a PhD student at ENAC (French Civil Aviation University). He has obtained telecommunication engineer diploma in 2014 and a Master of Science in wireless communication in 2016 at Politecnico di Torino, Italy. He is currently studying signal processing algorithms and integrity monitoring techniques adapted to the navigation in urban areas. His thesis is funded by ABBIA GNSS Technologies.

Axel Garcia Pena is a researcher/lecturer with the SIGnal processing and NAVigation (SIGNAV) research group of the TELECOM lab of ENAC (French Civil Aviation University), Toulouse, France. His research interests are GNSS navigation message demodulation, optimization and design, GNSS receiver design and GNSS satellite payload. He received his double engineer degree in 2006 in digital communications from SUPAERO and UPC, and his $\mathrm{PhD}$ in 2010 from the Department of Mathematics, Computer Science and Telecommunications of the INPT (Polytechnic National Institute of Toulouse), France.

Carl Milner is an Assistant Professor within the Telecom Lab at the Ecole Nationale de l'Aviation Civile. He has a Master degree in Mathematics from the University of Warwick, a PhD in Geomatics from Imperial College London and has completed the graduate trainee programme at the European Space Agency. His research interests include GNSS augmentation systems, integrity monitoring, air navigation and applied mathematics.

Bertrand EKAMBI graduated by a Master in Mathematical Engineering in 1999. Since 2000, he is involved in the main European GNSS projects: EGNOS and GALILEO. He is the founder manager of ABBIA GNSS Technologies, a French SME working on Space Industry, based in Toulouse, France.

\begin{abstract}
Stand-alone GNSS positioning is challenged to meet the applications' demanded accuracy and reliability in urban environments. In order to obtain an optimal and reliable position estimate using GNSS, it is necessary to have an accurate model of the pseudorange and pseudorange-rate error terms' distributions. Previous research contributions addressed the statistical characterization of the pseudorange measurements' multipath and noise error components in an urban environment by proposing a methodology to isolate the multipath error and the thermal noise component from the pseudorange measurement. The proposed methodology was adapted to include dual constellation measurements in the L1 band, GPS L1 C/A and Galileo E1 OS signal measurements. Moreover, a reliable classification of the signal reception conditions, Lion-of-Sight (LOS) and Non LOS (NLOS), was addressed.
\end{abstract}

The work conducted in this article consists first in further adapting the methodology proposed in the previous work to the pseduorange rate (Doppler frequency) measurement and second, in inspecting the benefits of the derived pseudorange and pseudorange-rate measurements' error component characterizations when applied on the PVT navigation solution. Finally, the analysis of the PVT estimation performance based on satellite measurement selection principle is presented.

\section{Introduction}

Global Navigation Satellite Systems (GNSS) is challenged to provide a continuous and accurate positioning service in harsh environments, such as urban environments, where the number of Line-of-Sight (LOS) visible satellites is limited [1]. Moreover, no orientation (or attitude) information is provided by GNSS standalone receivers in spite of being a very important factor for aiding the navigation in this kind of environments. One 
possible solution to cope with GNSS standalone receiver limitations, which is becoming a potential baseline platform for mass-market user devices, is to integrate an Inertial Measurement Unit (IMU), [1]. Such platforms aim at combining reasonably low-cost hardware with the provision of the high positioning accuracy, availability and reliability. More specifically, this kind of solution increases the quality of the PVT estimations by taking advantage of the complementary nature of GNSS and the inertial systems measurements. However, the major drawbacks of such a solution are the cost increase and the increased complexity of the PVT algorithms. However, nowadays, these drawbacks are mitigated due to the appearance of good performing low-cost IMUs, goodperforming low-cost chipsets and the development of fast GNSS receiver software-radio implementations.

Although IMU complements the weak points of GNSS in urban canyons, the low availability of LOS satellites and low-quality measurements (pseudoranges, Doppler frequency, carrier phase) make the hybrid system to still have to rely on GNSS measurements for correcting sensor drifts. Therefore, in order to obtain an optimal and reliable position estimate, it is necessary to have access to an accurate assessment of the GNSS measurements even in difficult environments; in other words, it is necessary to accurately model the pseudorange, doppler and carrier phase error distributions due to the presence of the multipath and NLOS received signal conditions.

Initial efforts to assess the quality of GNSS measurements in urban environments have already been done. In [2] and [3], a new enhanced characterization of the measurement errors' weighting in the Kalman Filter was approached. In [4] and [5], some different techniques based on detection, de-weighting and mitigation of multipath-affected measurement and/or measurement in Non Line-of-Sight signal conditions were developed. In [6], a study of the PVT estimation performance based on the combination and the selection of the joint GPS and GLONASS satellite measurements was conducted. Finally, in [7], a methodology to characterize the pseudorange measurement errors due to multipath for a GNSS receiver in a car in an urban environment was proposed. Applying this approach, a realistic model of the GPS + Galileo measurement error in an urban and sub-urban environment obtained by discriminating the multipath error contribution from the received signal Line-of-Sight (LOS) or Non Line-of-Sight (NLOS) situations was obtained. Moreover, an analysis of the measurement multipath and noise error distribution was provided as a function of the satellite elevation and the received signal $C / N_{0}$. This same methodology applied in [7] may be adapted to provide estimations of the Doppler frequency multipath error component distribution. By doing so, the measurements knowledge about multipath error distribution in an urban environment, pseudorange and pseudorange-rate, will be enhanced and, consequently, the GNSS standalone solution in urban environments, which is also used to correct sensor drifts, will be improved.

The aim of this paper is thus to propose a low-cost GNSS position solution algorithm which exploits the knowledge about the a priori multipath error component plus noise distributions of the pseudorange and pseudorange-rate measurements using the methodology provided in [7]. The expected benefits of such a solution are an increase of the PVT estimation accuracy and reliability without significant modification of the PVT solution algorithm.

Moreover, due to the more precise knowledges of the multipath and noise error joint characteristics and the possibility to differentiate between LOS and NLOS received signal conditions, an analysis of PVT estimation solutions based on an intelligent satellite measurement selection is proposed. This will be achieved by using the NLOS and LOS classification as a function of the received signal parameters (such as $\mathrm{C} / \mathrm{N}_{0}$ and elevation angle). The PVT solution is calculated by using:

- $\quad$ all available satellite measurements (LOS + NLOS conditions)

- $\quad$ satellite measurements only from satellites estimated to be in LOS conditions

- all available LOS-estimated satellite measurements and a smart selected group of NLOS-estimated satellite measurements

All available measurement could be used with their associated uncertainty (Gaussian distribution variance); in this case, the NLOS biased measurement could be mitigated due to the fine Gaussian distribution fitting associated to the measurement (as a function of the received signal C/NO and on the elevation angle) and even with the removal of the Gaussian mean on the measurement (since EKF requires centred gaussian noise).

The idea of using NLOS measurements instead of discarding them is twofold. One on side, the number of available measurements increases and thus the continuity of the solution should be improved. On the other hand, the final position estimation accuracy (and thus reliability) depends on the measurements' quality and on the geometry of the employed measurements (Dilution of Precision, DOP). Therefore, although the inclusion of NLOS measurements should decrease the measurements quality, the new geometry could improve the final DOP with a potential improvement of PVT accuracy. 
Third and last, following the principle explained in the previous point, it is possible that the best solution does not consists in blindly adding all NLOS measurements but only a specific subset should be used in order to ensure that the effect of adding them is beneficial to the final PVT accuracy.

The article is organized as follows:

- Section 1 introduces the pseudorange and pseudorange-rate measurement models and the pseudorangerate impairments due to multipath in urban environment.

- Section 2 recalls the multipath error plus thermal noise characterization, isolated from the pseudorange and the pseudorange-rate measurements.

- Section 3 describes the basic model for a stand-alone GNSS EKF solution and how to employ the doppler and pseudorange LOS/NLOS multipath error distribution in the stand-alone GNSS EKF solution to increase the estimation accuracy.

- Section 4 discusses the different methods to exploit LOS/NLOS satellite characterization knowledge in the stand-alone GNSS EKF solution to study the PVT estimation performances in different satellite conditions (LOS+NLOS, LOS, LOS + mix of NLOS).

- $\quad$ Finally, conclusions and future works are derived in Section 5.

\section{Pseudorange and Pseudorange-rate models}

The definition of pseudorange and pseudorange-rate measurement models for the GPS and Galileo constellations are presented in this section, introduncing all the error affecting the measurement.

The pseudorange measurement $\rho$ for a given satellite $i$, at epoch $k$, is modelled as follows:

$$
\rho^{i}[k]=\left(\tau^{i}[k]+\Delta t_{R T}^{i}[k]\right) c+\varepsilon_{T O T}^{i}[k]+M^{i}[k]+\eta^{i}[k]
$$

where:

- $\tau$ is the true signal propagation time from the satellite to the receiver on ground, expressed in seconds (s);

- $\Delta t_{R T}^{i}$ represents the error between the receiver $(R)$ and the transmitter satellite $(T)$ clock time, expressed in $\mathrm{s}$;

- $\quad$ c denotes the speed of light in meters/seconds $(\mathrm{m} / \mathrm{s})$;

- $\varepsilon_{T O T}^{i}$ denotes the propagation delays grouped in one term, expressed in $\mathrm{m}$;

- $\quad M^{i}$ denotes the error in $m$ due to multipath;

- $\eta^{i}$ represents the error, in $\mathrm{m}$, due to receiver AWG noise.

According to equation 1-1 and since this work focuses on a dual-constellation GPS/Galileo receiver platform, it is thus required to present the propagation delays for both GPS and Galileo constellations as:

The clock error term for GPS constellation, $\Delta t_{R T, G P S}^{i}$ is equal to:

$$
\Delta t_{R T, G P S}^{i}[k]=\Delta t_{R, G P S}[k]-\Delta t_{T, G P S}^{i}[k]=\left(t_{R}[k]-t_{G P S}[k]\right)-\left(t_{T, G P S}^{i}[k]-t_{G P S}[k]\right)
$$

where:

- $\quad t_{R}$ is the receiver time corresponding to epoch $k$ of the receiver's clock in s;

- $t_{T, G P S}^{i}$ is the satellite clock time based on the satellite clock in s;

- $t_{G P S}$ represents the GPS system time, computed by the GPS Master Control Station on ground as the weighted average of each GPS satellite time measurement, expressed also in s;

- $\quad c$ is the speed of light, in $\mathrm{m} / \mathrm{s}$.

The clock error term for GPS constellation, expressed in terms of range error, could be defined as

$$
\Delta t_{R T, G P S}^{i}[k] c=\Delta t_{R, G P S}[k] c-\Delta t_{T, G P S}^{i}[k] c=b_{R, G P S}[k]-b_{T, G P S}^{i}[k]
$$

- $\quad b_{R, G P S}$ is the receiver's clock bias with respect to GPS reference time expressed in $\mathrm{m} ; b_{T, G P S}^{i}$ is the i-th GPS satellite clock bias with respect to GPS reference time expressed in $\mathrm{m}$.

Performing the same steps for each clock term concerning the $i^{\text {th }}$ Galileo constellation, the following expression can be written:

$$
\Delta t_{R T, G A L}^{i}[k]=\Delta t_{R, G A L}[k]-\Delta t_{T, G A L}^{i}[k]=\left(t_{R}[k]-t_{G A L}[k]\right)-\left(t_{T, G A L}^{i}[k]-t_{G A L}[k]\right)
$$


with $t_{T, G A L}^{i}$ and $t_{G A L}$ representing the Galileo satellite clock time and the Galileo system time, respectively, expressed in s. The clock error term for Galileo constellation, expressed in terms of range error, could be defined as

$$
\Delta t_{R T, G A L}^{i}[k] c=\Delta t_{R, G A L}[k] c-\Delta t_{T, G A L}^{i}[k] c=b_{R, G A L}[k]-b_{T, G P S}^{i}[k]
$$

where, $b_{R, G A L}$ and $b_{T, G A L}^{i}$ are the receiver's clock bias and the i-th Galileo satellite clock bias with respect to GPS reference time expressed in $\mathrm{m}$.

However, dual constellation GNSS receiver can select only 1 GNSS time reference to express all the received measurements in order to have only 1 receiver clock time bias to estimate in the PVT solution. Therefore, taking as reference GPS time for example, expression $\Delta t_{R, G A L}[k]$ can be modified as:

$$
\begin{gathered}
\Delta t_{R, G A L}[k]=t_{R}[k]-t_{G A L}[k]=\left(t_{R}[k]-t_{G P S}[k]\right)+\left(t_{G P S}[k]-t_{G A L}[k]\right) \\
=\Delta t_{R, G P S}[k]+\Delta t_{G P S / G A L}[k]
\end{gathered}
$$

where $\Delta t_{G P S / G A L}$ is the inter-constellation clock offset expressed in seconds, , and, in terms of ranging error,

$$
\Delta t_{R, G A L}[k] c=\Delta t_{R, G P S}[k] c+\Delta t_{G P S / G A L}[k] c=b_{R, G P S}[k]+\delta \mathrm{b}[k]
$$

where $\delta \mathrm{b}$ is the inter-constellation clock bias expressed in meters.Based on the relations provided in 1-1 and 1-6, the code propagation delay for the $i^{t h}$ GPS satellite epoch $k$, is provided by:

$$
\begin{gathered}
\rho_{G P S}^{i}[k]=\left(\tau_{G P S}^{i}[k]+\Delta t_{R T, G P S}^{i}[k]\right) c+\varepsilon_{T O T, G P S}^{i}[k]+M_{G P S}^{i}[k]+\eta_{G P S}^{i}[k]= \\
=\left[\tau_{G P S}^{i}[k]+\left(\Delta t_{R, G P S}[k]-\Delta t_{T, G P S}^{i}[k]\right)\right] c+\varepsilon_{T O T, G P S}^{i}[k]+M_{G P S}^{i}[k]+\eta_{G P S}^{i}[k]= \\
=c \tau_{G P S}^{i}[k]+c \Delta t_{R, G P S}[k]-c \Delta t_{T, G P S}^{i}[k]+\varepsilon_{T O T, G P S}^{i}[k]+M_{G P S}^{i}[k]+\eta_{G P S}^{i}[k]= \\
=r_{G P S}^{i}[k]+b_{R, G P S}^{i}[k]-b_{T, G P S}^{i}[k]+\varepsilon_{T O T, G P S}^{i}[k]+M_{G P S}^{i}[k]+\eta_{G P S}^{i}[k]
\end{gathered}
$$

where the newly introduced terms for each epoch $k$ denote:

- $r_{G P S}^{i}=\sqrt{\left(x_{T, G P S}^{i}-x_{R}\right)^{2}+\left(y_{T, G P S}^{i}-y_{R}\right)^{2}+\left(z_{T, G P S}^{i}-z_{R}\right)^{2}}$ is the true geometrical range between the ith GPS satellite and the receiver for which $\left(x_{T, G P S}^{i}, y_{T, G P S}^{i}, z_{T, G P S}^{i}\right)$ denotes the i-th satellite position at transmit time, computed from the broadcast ephemeris file and expressed in the ECEF frame, and $\left(x_{R}, y_{R}, z_{R}\right)$ denotes the user position at receiver time, expressed in the ECEF frame.

The same relation also holds for the code propagation delay of the Galileo satellites, expressed as:

$$
\rho_{G A L}^{i}[k]=r_{G A L}^{i}[k]+b_{R, G P S}[k]-b_{T, G A L}^{i}[k]-\delta \mathrm{b}[k]+\varepsilon_{T O T, G A L}^{i}[k]+M_{G A L}^{i}[k]+\eta_{G A L}^{i}[k]
$$

For simplicity, $b_{R, G P S}=b_{R}$.

In order to observe the atmosphere-induced delays, the GPS and Galileo propagation delays can be written as:

$$
\begin{gathered}
\rho_{G P S}^{i}[k]=r_{G P S}^{i}[k]+b_{R}[k]-b_{T, G P S}^{i}[k]+I_{G P S}^{i}[k]+T_{G P S}^{i}[k]+M_{G P S}^{i}[k]+\eta_{G P S}^{i}[k] \\
\rho_{G A L}^{i}[k]=r_{G A L}^{i}[k]+b_{R}[k]-b_{T, G A L}^{i}[k]-\delta \mathrm{b}[k]+I_{G A L}^{i}[k]+T_{G A L}^{i}[k]+M_{G A L}^{i}[k]+\eta_{G A L}^{i}[k]
\end{gathered}
$$

where:

- $I_{G P S}^{i}$ and $I_{G A L}^{i}$ represent the ionosphere propagation delay contributions on the GPS and Galileo pseudorange measurements, respectively, expressed in $\mathrm{m}$;

- $T_{G P S}^{i}$ and $T_{G A L}^{i}$ represent the troposphere propagation delay contributions on the GPS and Galileo pseudorange measurements, respectively, expressed in $\mathrm{m}$.

The nominal pseudorange-rate measurement from a given constellation, $C, \dot{\rho_{C}}$, including the propagation delays and measurement errors, computed by the receiver for a given satellite $i$, at the epoch $k$ is modelled as follows:

$$
\begin{gathered}
\dot{\rho}_{C}^{i}[k]=\left(\dot{x}_{T, C}^{i}[k]-\dot{x}_{R, C}[k]\right) u_{C, x}^{i}+\left(\dot{y}_{T, C}^{i}[k]-\dot{y}_{R}[k]\right) u_{C, y}^{i}+\left(\dot{z}_{T, C}^{i}[k]-\dot{z}_{R}[k]\right) u_{C, Z}^{i}+ \\
\left(\dot{b}_{T, C}^{i}[k]-\dot{b}_{R}[k]\right)+\delta \mathrm{b}[k]+\dot{I}_{C}^{i}[k]+\dot{T}_{C}^{i}[k]+\dot{M}_{C}^{i}[k]+\dot{\eta}_{C}^{i}[k]
\end{gathered}
$$


where:

- $\left(\dot{x}_{T, C}^{i}, \dot{y}_{T, C}^{i}, \dot{z}_{T, C}^{i}\right)$ and $\left(\dot{x}_{R, C}, \dot{y}_{R, C}, \dot{z}_{R, C}\right)$ represent the i-th satellite of C constellation (GPS or Galileo) and user's velocity vectors in the ECEF reference frame, respectively, expressed in $\mathrm{m} / \mathrm{s}$;

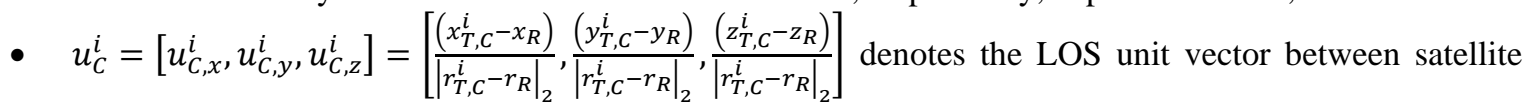
and receiver;

- $\quad \dot{b}_{T}^{i}$ is the satellite clock drift;

- $\quad \dot{b}_{R}$ is the receiver clock drift;

- $\delta \dot{\mathrm{b}}$ is the inter-constellation clock drift, which is assumed to be negligible with the respect to the other terms;

- $\quad i^{i}$ is the delay drift of the error due to the presence of the ionosphere;

- $\quad \dot{T}^{i}$ is the delay drift of the error due to the presence of the troposphere;

- $\dot{M}^{i}$ is the rate of change of the error due to the presence of the multipath;

- $\quad \dot{\eta}^{i}$ denotes the rate of change of the receiver's thermal noise effect.

One of the errors affecting the pseudorange-rate measurement is the error introduced by the multipath effect, which is the dominant error source in the urban environment. The multipath is difficult to remove or at least mitigate by low-cost equipment and is hard to model since the multipath effect depends on the environment and the emitter's and receiver's velocities. The next section is devoted to the isolation of the multipath and the noise error components from the pseudorange-rate measurements.

\section{Doppler frequency Multipath and Noise Joint Error Component}

In this section, the methodology used in [7]:

- to isolate the multipath error and the noise from the pseudorange measurement and,

- to characterize it in terms of the receiver $C / N_{0}$ and LOS/NLOS received signal conditions

is adapted to the pseudorange-rate measurements.

In section 2.1, the methodology applied to isolate the pseudorange-rate multipath and noise joint error isolation will be recalled, while in section 2.2 , the overall characterization results, which comprises the pseudorange and the pseudorange-rate multipath and noise error distributions, are discussed.

\subsection{Doppler frequency Multipath and Noise Joint Error Component Isolation}

The proposed method consists in isolating, as much as possible, the multipath error component from the other pseudorange-rate measurement terms. The procedures employed for the removal of the different terms depends on the nature of each term:

- The satellite-to-receiver range-rate can be easily subtracted if the receiver and satellite position are known;

- The removal of all atmospheric and satellite-dependent elements can be obtained by differencing the pseudorange-rate measurements with the measurements of a nearby reference station;

- The receiver clock drift can be estimated first and removed later.

In this section, the method is adapted to the pseudorange-rate measurements. The three steps are summarized below:

1. To obtain a pseudorange-rate residual which contains only the pseudorange-rate error, also called "rangerate-free" component, removing the true range-rate component from the range-rate measurement. The same can be done to obtain "range-rate-free" reference station pseudorange-rate residuals.

2. To remove the impairments from the vehicle receiver pseudorange-rate residual which are common to the reference station ones. The removal is achieved by differencing the user "range-rate free" measurement from the "range-rate free" reference station pseudorange-rate residual. The differential measurement can be simplified into (2-1) as justified in [7]:

$$
\dot{\epsilon}^{i}(t) \approx \dot{b}(t)+M N_{u}^{i}(t)
$$

where 
- the reference station Doppler multipath error component as well as the residual atmospheric errors and residual ephemeris errors are assumed to be negligible with respect to $\dot{M}_{u}^{i}(t)$;

- Multipath error and thermal noise are modelled as a single error term, $M N_{u}^{i}(t)$ since the reference station thermal noise component is considered to be negligible compare to receiver noise and multipath in urban environments;

- the vehicle-reference station receiver clock drift difference, are estimated together as a unique term called clock drift term, $\dot{b}_{u}(t)$.

3. To isolate each individual multipath error component in addition to the thermal noise components, $\widehat{M N}_{u}^{i}(t)$, [7], from the clock drift term, $\dot{b}(t)$. The isolation process is conducted by

a. Estimating $\dot{b}(t)$ from the pseudorange-rate residual difference terms;

b. Removing/subtracting the estimated clock drift term, $\dot{b}(t)$, from each pseudorange-rate residual difference, $\dot{\epsilon}^{i}(t)$, to estimate each $\widehat{M N}_{u}^{i}(t)$. In order to estimate $\dot{b}_{u}(t)$, a lowpass frequency filter could be applied to the any pseudorange-rate residual difference term since this term has its power spectrum density around the lowest frequencies among all the pseudorange-rate residual difference terms [7]. The lowpass filter is a first-order Butterworth with a cutoff frequency equal to $0.01 \mathrm{~Hz}$, as showed in Figure 1.

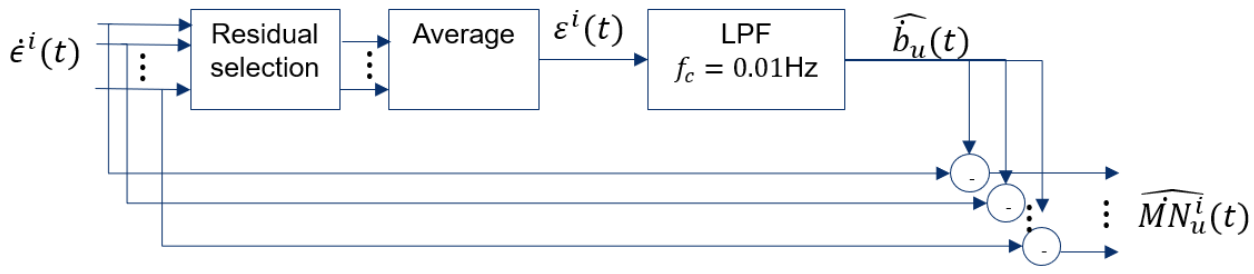

Figure 1 - Isolation of multipath and thermal noise error component from clock drift component

\subsection{Multipath and Noise Joint Error Component Characterization}

This section discusses the characteristics of the Multipath and noise joint error characterization isolated from the pseudorange [7] and the pseudorange-rate, having applied the methodology in section 2.1.

Since the multipath and noise isolation and characterization is provided in [7], this work is focused on the results isolated from the pseudorange-rate. Once the multipath error plus thermal noise component, $\widehat{M N}_{u}^{i}$, is isolated, the multipath plus noise joint characterization process can be applied in order to obtain a mathematical model, which defines the multipath plus noise joint error component statistical behaviour. The section 2.2.1 describes the user equipment and the experimental setup used to make the pseudorange error characterization in [7]. The section 2.2.2 successively shows the results of Multipath and noise joint error characterization isolated from the pseudorange [7] and the pseudorange-rate obtained using the experimental data as applied in 2.2.1.

\subsubsection{Experimental setup}

The equipment used during the data collection campaign consists of the following devices:

a. U-Blox M8T: GPS receiver.

b. Novatel \& Span GPS/GLONASS receiver.

c. CNES Toulouse reference station receiver

d. IDS uEye Camera with fish-eye lens

e. Laptop used for the experiment

f. ENAC test vehicle used to do the experimental data campaign.

The role of the uBlox M8T receiver in the experiment is to be the low-cost "mass-market" receiver working in the L1 frequency band which collects the data to be analysed. The role of the NovAtel SPAN receiver is to obtain a very precise trajectory of the car during the data collection campaign which will be used as the true position of the receiver at any instant of time. Moreover, the SPAN receiver is also responsible for providing the vehicle heading information which can be extrapolated to the test receiver antenna using the known level-arm between IMU and the antenna. The SPAN receiver accuracy is at the decimetre-level or better. 


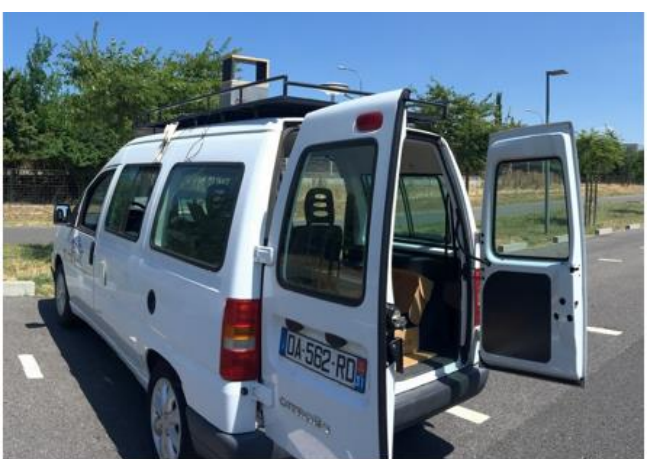

Figure 2- Picture of the vehicle used for the experiment



Figure 3- Rooftop of the vehicle, showing the uBlox, NovAtel antennas and the fish-eye camera

The reference station used in the experiment is the one located in the Toulouse site of the Centre National d'Etudes Spatiales (CNES). Figure 2, Figure 3, show the test vehicle and the roof of the vehicle. Inside the van a small laboratory allows to work with the laptops and take the data campaign under control. The role of the fish-eye camera consists in taking the pictures of the vehicle sky-environment in synchronization with the U-Blox estimated time. The fish-eye camera used in this study consists of a fish-eye lens attached to an IDS company CMOS sensor. The camera has a field angle greater than $180^{\circ}$ and captures images of the entire perimeter with an elevation angle range going from $0^{\circ}$ to $90^{\circ}$. The captured images are in a greyscale and have a resolution of $1280 \times 1024$ pixes.

The trajectory followed in Toulouse urban area is divided in three different sections, ordered in the chronologically sequence.

The selected location for the data campaign was Toulouse urban area. The location was chosen in order to have a representation of different types of obstacles and different LOS/NLOS scenarios:

- $\quad$ the city center, with large and small streets around tall buildings and areas including bunch of trees;

- $\quad$ the suburbs; large and small streets in presence of small buildings; open areas close to the river.

\subsubsection{Multipath and noise joint distribution characterization results}

The $\widehat{M N}{ }_{u}^{i}$ first is characterized and organized in several received signal $C / N_{0}$ bands [7]. After applying the image processing and the signal processing LOS/NLOS characterization, [7] the components are classified into different groups with the same characteristics where each group represents a specific received satellite signal reception condition (Non Line-of-Sight, Line-of-Sight, mix of LOS and NLOS).

As stated in [7], the pseudorange multipath error and noise components joint model is characterized by the Empirical probability density function, $\operatorname{PDF}\left(\widehat{M N}_{u}^{i}\right)$. From the Empirical probability density function could be extracted also the sample mean $\mu\left(\widehat{M N}_{u}^{i}\right)$ and the sample standard deviation, $\sigma\left(\widehat{M N}_{u}^{i}\right)$. As an example, a set of results regarding the pseudorange multipath and noise error distribution is presented in Table 1, obtained from the dataset used in [7].

A set of results regarding the pseudorange multipath and noise error distribution is presented in Table 1. Table 1 provides the sample mean, sample standard deviations and receiver signal conditions of $\widehat{M N}_{u}^{i}$ results obtained from the dataset used in [7]. The results are divided in three different sections depending on the LOS/NLOS received signal conditions, as stated in [7]. For low $C / N_{0}$ bands, below $35 \mathrm{~dB}-\mathrm{Hz}$, the error distributions are classified as NLOS signal condition error distributions. The resulting error distributions are positive-biased, nonGaussian and non-symmetric, featured by high standard deviations, which are inversely related to the $C / N_{0}$. For the $C / N_{0}$ bands higher than $38 \mathrm{~dB}-\mathrm{Hz}$, the distributions are depending on satellite measurement errors classified as LOS signal conditions. The distributions are zero-mean Gaussian shaped with a standard deviation inversely related to the $C / N_{0}$ decrease. The $36 / 38 \mathrm{~dB}-\mathrm{Hz}$ band contains pseudorange errors isolated from a mix of NLOS and LOS satellites.

Then, the methodology proposed is adapted to the characterization of the pseudorange-rate measurements, the results are showed in Table 2. The pseudorange-rate multipath error and noise components joint model is 
characterized by the Empirical probability density function, $P D F\left(\widehat{M N}{ }_{u}^{i}\right)$. From the empirical probability density function, could be extracted the sample mean $\mu\left(\widehat{M N}_{u}^{i}\right)$ and the sample standard deviation, $\sigma\left(\widehat{M N}_{u}^{i}\right)$.

Some results taken from the table are presented next. Figure 4 corresponds to the LOS multipath error component PDF in the $45-50 \mathrm{~dB}-\mathrm{Hz} \mathrm{C} \backslash \mathrm{N}_{0}$ range. The multipath error component has a Gaussian shape centred in 0. Finally, Figure 5 corresponds to the NLOS multipath error component PDF in the $20-25 \mathrm{~dB}-\mathrm{Hz} \mathrm{C} \backslash \mathrm{N}_{0}$ range. The PDF seems to be a symmetric and zero-mean distribution. The PDF's positive and negative tails seem to be higher than the example presented before. This phenomenon is probably due to the presence of the Doppler spreading and, mainly, due to a higher level of thermal noise and a higher number of multipath rays.

\begin{tabular}{|c|c|c|c|}
\hline $\mathrm{C} / \mathrm{N}_{0}$ range & $\mu\left(\widehat{M N}_{u}^{i}\right)[\mathrm{m}]$ & $\left.\sigma(\widehat{M N})_{u}^{i}\right)[\mathrm{m}]$ & RX signal condition \\
\hline $32 / 34(\mathrm{~dB}-\mathrm{Hz})$ & 9,5608 & 19,95 & \multirow{2}{*}{ Definition of NLOS conditions } \\
\hline $34 / 36(\mathrm{~dB}-\mathrm{Hz})$ & 6,6890 & 15,98 & \\
\hline $36 / 38(\mathrm{~dB}-\mathrm{Hz})$ & 2,9046 & 12,97 & Mix LOS and NLOS conditions \\
\hline $38 / 40(\mathrm{~dB}-\mathrm{Hz})$ & 0,9005 & 0.9163 & \multirow{3}{*}{ Definition of LOS conditions } \\
\hline $40 / 42(\mathrm{~dB}-\mathrm{Hz})$ & $-0,0246$ & 0.2632 & \\
\hline $42 / 44(\mathrm{~dB}-\mathrm{Hz})$ & 0,0047 & 0.0581 & \\
\hline
\end{tabular}

Table 1 - Table containing the pseudorange multipath error component pdf's mean values and standard deviations characterized by a different $C / N_{0}$ range

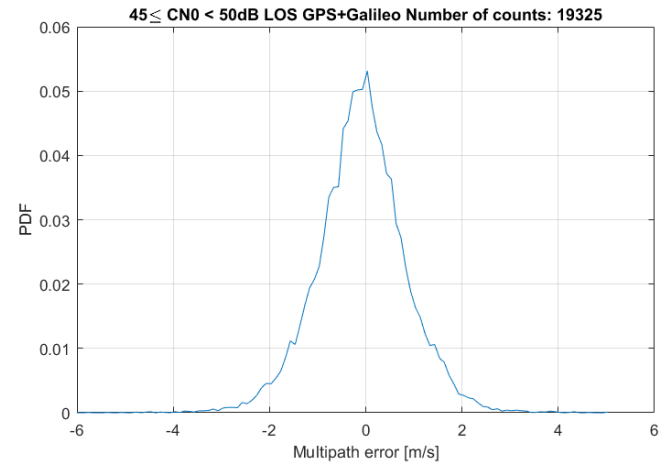

Figure 4 - Pdf of pseudorange-rate multipath and noise joint error component in the $45-50 \mathrm{~dB}-\mathrm{Hz} \mathrm{C} \backslash \mathrm{N}_{0}$ band.



Figure 5 - Pdf of pseudorange-rate multipath and noise joint error component in the $30-35 \mathrm{~dB}-\mathrm{Hz} C \backslash N_{0}$ band. GPS constellation cas

\begin{tabular}{|c|c|c|c|}
\hline $\mathrm{C} / \mathrm{N}_{0}$ range & $\mu\left(\widehat{\operatorname{MN}}_{u}^{i}\right)[\mathrm{m}]$ & $\sigma\left(\widehat{M N_{u}^{i}}\right)[\mathrm{m}]$ & $\mathrm{RX}$ signal condition \\
\hline $32 / 34(\mathrm{~dB}-\mathrm{Hz})$ & 0,0820 & 1,4343 & \multirow{2}{*}{ Definition of NLOS conditions } \\
\hline $34 / 36(\mathrm{~dB}-\mathrm{Hz})$ & 0,0457 & 1,2233 & \\
\hline $36 / 38(\mathrm{~dB}-\mathrm{Hz})$ & 0,0348 & 1,1300 & Mix LOS and NLOS conditions \\
\hline $38 / 40(\mathrm{~dB}-\mathrm{Hz})$ & $-0,0078$ & 0,6924 & \multirow{3}{*}{ Definition of LOS conditions } \\
\hline $40 / 42(\mathrm{~dB}-\mathrm{Hz})$ & $-0,0434$ & 0,4948 & \\
\hline $42 / 44(\mathrm{~dB}-\mathrm{Hz})$ & $-0,0464$ & 0,3675 & \\
\hline
\end{tabular}


Table 2 - Table containing the pseudorange-rate multipath error component pdf's mean values and standard deviations characterized by a different $C / N_{0}$ range

\section{Proposed Extended Kalman Filter}

This chapter provides a clear description of the proposed GNSS dual-constellation Extended Kalman Filter implemented in this work, focusing on the state space description and the measurement processing computation. Successively, a possible improvement of the EKF, based on the knowledges presented in section 2, is discussed.

The first section, 3.1, provides the State Space description. The next sections, 3.2 and 3.3, provide the Extended Kalman Filter Measurement model used in this work with the estimator computational steps, and the Measurement noise covariance matrix, respectively.

Finally, a modification of the Measurement noise covariance matrix based on the multipath and noise error joint distribution knowledges in urban environment is proposed in section 3.4, and applied to the EKF model in section 3.5. As a consequence, the performances of the two EKF models, basic one and modified one, are provided in section 3.5 .

\subsection{EKF State Space description}

The state vector, at a given epoch $k$, is equal to:

$$
x_{k}=(p, v, a, b, \dot{b}, \delta b)=(x, y, z, \dot{x}, \dot{y}, \dot{z}, \ddot{x}, \ddot{y}, \ddot{z}, b, \dot{b}, \delta b)
$$

where:

- $\quad p=[x, y, z]^{T}$ represents the 3-D user's position expressed in the ECEF frame in $\mathrm{m}$

- $\quad v=[\dot{x}, \dot{y}, \dot{z}]^{T}$ represents the 3-D user's speed expressed in the ECEF frame in $\mathrm{m} / \mathrm{s}$

- $\quad a=[\ddot{x}, \ddot{y}, \ddot{z}]^{T}$ represents the 3-D user's speed expressed in the ECEF frame in $\mathrm{m} / \mathrm{s}^{2}$

- $[b, \dot{b}]^{T}$ denotes the user's clock bias in $\mathrm{m}$, and drift in $\mathrm{m} / \mathrm{s}$, respectively.

- $\delta b$ is the GPS-to-Galileo inter-constellation bias, in $\mathrm{m}$.

and the state covariance matrix is equal to:

$$
P_{0}=\left(\begin{array}{cccccccccccc}
\sigma_{x}^{2} & 0 & 0 & 0 & 0 & 0 & 0 & 0 & 0 & 0 & 0 & 0 \\
0 & \sigma_{y}^{2} & 0 & 0 & 0 & 0 & 0 & 0 & 0 & 0 & 0 & 0 \\
0 & 0 & \sigma_{z}^{2} & 0 & 0 & 0 & 0 & 0 & 0 & 0 & 0 & 0 \\
0 & 0 & 0 & \sigma_{\dot{x}}^{2} & 0 & 0 & 0 & 0 & 0 & 0 & 0 & 0 \\
0 & 0 & 0 & 0 & \sigma_{\dot{y}}^{2} & 0 & 0 & 0 & 0 & 0 & 0 & 0 \\
0 & 0 & 0 & 0 & 0 & \sigma_{\dot{z}}^{2} & 0 & 0 & 0 & 0 & 0 & 0 \\
0 & 0 & 0 & 0 & 0 & 0 & \sigma_{\ddot{x}}^{2} & 0 & 0 & 0 & 0 & 0 \\
0 & 0 & 0 & 0 & 0 & 0 & 0 & \sigma_{\ddot{y}}^{2} & 0 & 0 & 0 & 0 \\
0 & 0 & 0 & 0 & 0 & 0 & 0 & 0 & \sigma_{\ddot{z}}^{2} & 0 & 0 & 0 \\
0 & 0 & 0 & 0 & 0 & 0 & 0 & 0 & 0 & \sigma_{b, G P S}^{2} & 0 & 0 \\
0 & 0 & 0 & 0 & 0 & 0 & 0 & 0 & 0 & 0 & \sigma_{\dot{b}, G P S}^{2} & 0 \\
0 & 0 & 0 & 0 & 0 & 0 & 0 & 0 & 0 & 0 & 0 & \sigma_{\delta b}^{2}
\end{array}\right)
$$

where

- $\sigma_{r}^{2}=\left[\sigma_{x}^{2}, \sigma_{y}^{2}, \sigma_{z}^{2}\right]$ is the initial position error variance,

- $\sigma_{v}^{2}=\left[\sigma_{\dot{x}}^{2}, \sigma_{\dot{y}}^{2}, \sigma_{\dot{z}}^{2}\right]$ is the initial speed error variance,

- $\sigma_{a}^{2}=\left[\sigma_{\ddot{x}}^{2}, \sigma_{\ddot{y}}^{2}, \sigma_{\ddot{z}}^{2}\right]$ is the initial acceleration error variance,

- $\sigma_{b, G P S}^{2}$ is the initial GPS clock bias error variance,

- $\sigma_{\dot{b}, G P S}^{2}$ is the initial GPS clock drift error variance,

- $\sigma_{\delta b}^{2}$ is the initial GPS-to-Galileo inter-constellation bias error variance. 
The first stage of the EKF estimation, referred to as the "state prediction", corresponds to the forward time projection of the state vector $x_{k}^{-}$and state covariance matrix $P_{k}^{-}$prior to the measurement inclusion, is performed in two steps:

1. State prediction: Propagation of the state vector estimate through time using

$$
x_{k}^{-}=\Phi_{k-1} x_{k-1}^{+}
$$

2. State Covariance Matrix Prediction

$$
P_{k}^{-}=\Phi_{k-1} P_{k-1}^{+} \Phi_{k-1}^{T}+Q_{k-1}
$$

where $\Phi$ is the phase transition matrix, 3-5, and $Q$ the system noise covariance matrix, 3-6.

The discrete state transition matrix $\Phi_{k}$ is equal to [8]:

$$
\Phi_{\mathrm{k}-1}=\left(\begin{array}{cccccccccccc}
1 & 0 & 0 & \tau_{s} & 0 & 0 & \frac{\tau_{s}^{2}}{2} & 0 & 0 & 0 & 0 & 0 \\
0 & 1 & 0 & 0 & \tau_{s} & 0 & 0 & \frac{\tau_{s}^{2}}{2} & 0 & 0 & 0 & 0 \\
0 & 0 & 1 & 0 & 0 & \tau_{s} & 0 & 0 & \frac{\tau_{s}^{2}}{2} & 0 & 0 & 0 \\
0 & 0 & 0 & 1 & 0 & 0 & \tau_{s} & 0 & 0 & 0 & 0 & 0 \\
0 & 0 & 0 & 0 & 1 & 0 & 0 & \tau_{s} & 0 & 0 & 0 & 0 \\
0 & 0 & 0 & 0 & 0 & 1 & 0 & 0 & \tau_{s} & 0 & 0 & 0 \\
0 & 0 & 0 & 0 & 0 & 0 & 1 & 0 & 0 & 0 & 0 & 0 \\
0 & 0 & 0 & 0 & 0 & 0 & 0 & 1 & 0 & 0 & 0 & 0 \\
0 & 0 & 0 & 0 & 0 & 0 & 0 & 0 & 1 & 0 & 0 & 0 \\
0 & 0 & 0 & 0 & 0 & 0 & 0 & 0 & 0 & 1 & \tau_{s} & 0 \\
0 & 0 & 0 & 0 & 0 & 0 & 0 & 0 & 0 & 0 & 1 & 0 \\
0 & 0 & 0 & 0 & 0 & 0 & 0 & 0 & 0 & 0 & 0 & 1
\end{array}\right)
$$

where $\tau_{s}$ is the time constant, equal to 1 second.

While, the system noise covariance matrix is equal to [8]:



where,

- $S_{a}$ is the acceleration PSD matrix resolved about the axes of ECEF frame, These depends on the dynamic of the application. A suitable value used for the data campaign under exam [1] is $1 \mathrm{~m}^{2} \mathrm{~s}^{-3}$;

- $S_{c k, \phi}$ is the receiver clock phase-drift PSD, typical value for a TCXO is $0.01 \mathrm{~m}^{2}$ [1];

- $S_{c k, f}$ is the receiver clock frequency-drift PSD, typical value for a TCXO is $0.04 \mathrm{~m}^{2}$ [1];

- $S_{\text {const }}$ is the inter-constellation GPS-to-Galileo PSD, which is considered small compared to the $S_{c k, \phi}$;

- $\quad$ The used value is equal to $0.0001 \mathrm{~m}^{2}$, [9]; 
- $\tau_{s}$ is the time constant, which is equal to 1 second.

\subsection{Measurement Model}

The measurement vector is based on the pseudorange and pseudorange-rate measurements obtained from a given satellite at a given epoch $k$, as showed in 1-10,1-11, and 1-12. Moreover, the pseudorange and pseudorange-rate measurements are corrected before being used by the EKF in order to remove the satellite clock bias, and ionosphere and troposphere error components [10]. After the application of the error correction models to the GPS and Galileo pseudorange and pseudorange-rate measurements, respectively, a resultant ionosphere residual appears in the received observations. The same happens after the application of a tropospheric and satellite clock correction models. Therefore, the corrected pseudorange and pseudorange-rate measurement models for both GPS and Galileo satellites remain the same as in the above section, but with the appearance of residuals for the ionosphere, troposphere and satellite clock terms instead of the initial terms:

where:

$$
\begin{gathered}
\tilde{\rho}_{G P S}^{i}[k]=r_{G P S}^{i}[k]+b_{R}[k]-\tilde{b}_{T, G P S}^{i}[k]+\tilde{I}_{G P S}^{i}[k]+\tilde{T}_{G P S}^{i}[k]+M_{G P S}^{i}[k]+\eta_{G P S}^{i}[k] \\
\tilde{\rho}_{G A L}^{i}[k]=r_{G A L}^{i}[k]+b_{R}[k]-\tilde{b}_{T, G A L}^{i}[k]+\delta b[k]+\tilde{I}_{G A L}^{i}[k]+\tilde{T}_{G A L}^{i}[k]+M_{G A L}^{i}[k]+\eta_{G A L}^{i}[k]
\end{gathered}
$$

- $\quad \tilde{b}_{T, C}^{i}$ denotes the GPS and Galileo satellite clock bias residual after correction expressed in $\mathrm{m}$

- $\quad \tilde{I}_{C}^{i}$ denotes the GPS and Galileo ionosphere residuals affecting the i-th satellite after the application of the Klobuchar an Ne-quick ionosphere correction models, respectively, expressed in $\mathrm{m} / \mathrm{s}$

- $\quad \tilde{T}_{C}^{i}$ denotes the GPS and Galileo troposphere residuals affection the i-th satellite after the application of the troposphere UNB-3 correction model, respectively, expressed in m/s

A similar relation can be written for the corrected $C$ constellation pseudorange-rate measurements as follows:

where:

$$
\begin{gathered}
\tilde{\dot{\rho}}_{C}^{i}[k]=\left(\dot{x}_{T, C}{ }^{i}[k]-\dot{x}_{R, C}[k]\right) u_{R T, x, C}^{i}+\left(\dot{y}_{T, C}{ }^{i}[k]-\dot{y}_{R}[k]\right) u_{R T, y, C}^{i}+\left(\dot{z}_{T, C}{ }^{i}[k]-\dot{z}_{R}[k]\right) u_{R T, z, C}^{i} \\
+\left(\dot{b}_{R}[k]-\tilde{\dot{b}}_{T, C}^{i}[k]\right) c+\tilde{I}_{C}^{i}[k]+\tilde{\dot{T}}_{C}^{i}[k]+\dot{M}_{C}^{i}[k]+\dot{\eta}_{C}^{i}[k]
\end{gathered}
$$

- $\quad \tilde{\dot{b}}_{T, C}^{i}$ denotes the GPS and Galileo satellite clock residual drift affecting the $i$-th pseudorange-rate measurement, assumed to be negligible with the respect to the other components.

- $\quad \tilde{I}_{C}^{i}$ denotes the GPS and Galileo ionosphere residual rate affecting the $i$-th pseudorange-rate measurement

- $\quad \tilde{\dot{T}}_{C}^{i}$ denotes the GPS and Galileo troposphere residual rate affecting the $i$ - $t h$ pseudorange-rate measurement

Finally, the corrected pseudorange and pseudorange-rate measurements obtained from the $N$ different visible satellites are included in the "corrected" measurement vector $\tilde{z}_{k}$ at a given epoch $k$, as input of the navigation filter provided by:

$$
\tilde{z}_{k}=\left\{\tilde{\rho}_{C}^{1}[k], \tilde{\rho}_{C}^{2}[k], \tilde{\rho}_{C}^{3}[k], \ldots, \tilde{\rho}_{C}^{\mathrm{N}}[k] \mid \tilde{\dot{\rho}}_{C}^{1}[k], \tilde{\dot{\rho}}_{C}^{2}[k], \tilde{\dot{\rho}}_{C}^{3}[k], \ldots, \tilde{\dot{\rho}}_{C}^{\mathrm{N}}[k]\right\}
$$

The dual-constellation observation matrix $H_{k}$, used in the proposed EKF, 3-10, is constructed by combining the partial derivatives terms concerning the pseudorange and pseudorange-rate measurements for the GPS and Galileo locked satellites: 


$$
H_{k}=\left(\begin{array}{cccccccccccc}
-u_{x, G P S}^{1} & -u_{y, G P S}^{1} & -u_{z, G P S}^{1} & 0 & 0 & 0 & 0 & 0 & 0 & 1 & 0 & 0 \\
-u_{x, G P S}^{2} & -u_{y, G P S}^{2} & -u_{z, G P S}^{2} & 0 & 0 & 0 & 0 & 0 & 0 & 1 & 0 & 0 \\
\ldots & \ldots & \ldots & \ldots & \ldots & \ldots & \ldots & \ldots & \ldots & \ldots & \ldots & \ldots \\
-u_{x, G P S}^{N} & -u_{y, G P S}^{N} & -u_{z, G P S}^{N} & 0 & 0 & 0 & 0 & 0 & 0 & 1 & 0 & 0 \\
-u_{x, G A L}^{1} & -u_{y, G A L}^{1} & -u_{z, G A L}^{1} & 0 & 0 & 0 & 0 & 0 & 0 & 1 & 0 & 1 \\
-u_{x, G A L}^{2} & -u_{y, G A L}^{2} & -u_{z, G A L}^{2} & 0 & 0 & 0 & 0 & 0 & 0 & 1 & 0 & 1 \\
\ldots & \ldots & \ldots & \ldots & \ldots & \ldots & \ldots & \ldots & \ldots & \ldots & \ldots & \ldots \\
-u_{x, G A L}^{N} & -u_{y, G A L}^{N} & -u_{z, G A L}^{N} & 0 & 0 & 0 & 0 & 0 & 0 & 1 & 0 & 1 \\
0 & 0 & 0 & -u_{x, G P S}^{1} & -u_{y, G P S}^{1} & -u_{z, G P S}^{1} & 0 & 0 & 0 & 0 & 1 & 0 \\
0 & 0 & 0 & -u_{x, G P S}^{2} & -u_{y, G P S}^{2} & -u_{z, G P S}^{2} & 0 & 0 & 0 & 0 & 1 & 0 \\
\ldots & \ldots & \ldots & \ldots & \ldots & \ldots & \ldots & \ldots & \ldots & \ldots & \ldots & \ldots \\
0 & 0 & 0 & -u_{x, G S S}^{N} & -u_{y, G P S}^{N} & -u_{z, G P S}^{N} & 0 & 0 & 0 & 0 & 1 & 0 \\
0 & 0 & 0 & -u_{x, G A L}^{1} & -u_{y, G A L}^{1} & -u_{z, G A L}^{1} & 0 & 0 & 0 & 0 & 1 & 0 \\
0 & 0 & 0 & -u_{x, G A L}^{2} & -u_{y, G A L}^{2} & -u_{z, G A L}^{2} & 0 & 0 & 0 & 0 & 1 & 0 \\
\ldots & \ldots & \ldots & \ldots & \ldots & \ldots & \ldots & \ldots & \ldots & \ldots & \ldots & \ldots \\
0 & 0 & 0 & -u_{x, G A L}^{N} & -u_{y, G A L}^{N} & -u_{z, G A L}^{N} & 0 & 0 & 0 & 0 & 1 & 0
\end{array}\right)
$$

\subsection{Measurement Noise covariance Matrix}

The measurement noise covariance matrix at given epoch $k, R_{k}$, models the noise-like errors on the pseudorange and pseudorange-rate measurements, such as tracking errors, multipath variations, and satellite clock noise. In the Kalman Filter, the measurement errors are modelled as White Gaussian noise errors, therefore, $R_{k}$ is modelled as diagonal matrix where the diagonal factors are the measurement error variances:

$$
R_{k}=\left(\begin{array}{cccccc}
\sigma_{\rho_{C}^{1}}^{2}[k] & 0 & 0 & 0 & 0 & 0 \\
0 & \sigma_{\rho_{C}^{2}}^{2}[k] & 0 & 0 & 0 & 0 \\
\ldots & \ldots & \ldots & \ldots & \ldots & \ldots \\
0 & 0 & \sigma_{\rho_{C}^{N}}^{2}[k] & 0 & 0 & 0 \\
0 & 0 & 0 & \sigma_{\dot{\rho}_{C}^{1}}^{2}[k] & 0 & 0 \\
0 & 0 & 0 & 0 & \sigma_{\dot{\rho}_{C}^{2}}^{2}[k] & 0 \\
\ldots & \ldots & \ldots & \ldots & \ldots & \ldots \\
0 & 0 & 0 & 0 & 0 & \sigma_{\dot{\rho}_{C}^{N}}^{2}[k]
\end{array}\right)
$$

Depending on the different satellite $i$, from the different constellation, $C$, at a given epoch $k$, the theoretical pseudorange measurement error variances are equal to:

$$
\sigma_{\rho_{C}^{i}}^{2}[k]=\left(\sigma_{C, D L L^{i}}^{2}[k]+\sigma_{C, M P}^{2}[k]\right)+\sigma_{C, U R A^{i}}^{2}[k]+\sigma_{C, I^{i}}^{2}[k]+\sigma_{C, T^{i}}^{2}[k]
$$

where,

- $\sigma_{D L L}^{2}$ is the variance of the DLL tracking error due to the AWGN noise at epoch $k$

- $\sigma_{M P}^{2}$ is the variance of the DLL tracking error due to multipath at epoch $k$

- $\sigma_{U R A}^{2}$ is the variance communicated by the User Range Accuracy at epoch $k$

- $\sigma_{I}^{2}$ is the ionosphere residual error variance after Klobuchar ionosphere model correction at epoch $k$

- $\sigma_{T}^{2}$ is the variance of the troposphere residual error after UNB-3 troposphere model correction at epoch $k$.

while the theoretical pseudorange-rate measurement error variances are equal to:

$$
\sigma_{\dot{\rho}_{C}^{i}}^{2}[k]=\left(\sigma_{C, F L L^{i}}^{2}[k]+\sigma_{C, m p^{i}}^{2}[k]\right)
$$

- $\sigma_{F L L}^{2}$ is the variance of the FLL tracking error due to the AWGN noise at epoch $k$;

- $\sigma_{\text {mip }}^{2}$ is the variance of the FLL tracking error due to multipath at epoch $k$.

\subsection{Modified Measurement Noise Covariance Matrix}


The basic Measurement Noise Covariance Matrix, presented in 3-11, could be modified exploiting the knowledges of the realistic multipath and noise joint error characteristics discussed in the section 2.2.

The theoretical pseudorange and pseudorange-rate error variances, 3-12, 3-13, could be modified substituting the theoretical model of the DLL and FLL tracking error due to the AWGN noise and the multipath:

- $\quad \sigma_{C, D L L^{i}}^{2}[k]+\sigma_{C, M P^{i}}^{2}[k]$ for the pseudorange measurement variances,

- $\quad \sigma_{C, F L L^{i}}^{2}[k]+\sigma_{C, m p^{i}}^{2}[k]$ for the pseudorange-rate measurement variances

with the realistic pseudorange and pseudorange-rate multipath plus noise joint error distribution variances obtained through Table 1 and Table 2, $\sigma\left(\widehat{M N}_{u}^{i}\right)$ and $\sigma\left(\widehat{M N}_{u}^{i}\right)$.

As assumed in Section 2.2, the pseudorange error distribution is clearly divided in three different sections depending on the received signal $C / N_{0}$ and the LOS/NLOS received signal conditions. It is stated that for high $C / N_{0}$, when the distribution is obtained by isolated errors coming from signal defined to be in LOS conditions, the error model is Gaussian and zero-mean, while in case of signals defined in NLOS, the distribution is nonGaussian, non-symmetric and positive biased. However, the basic Kalman filter is an optimal estimator with the assumption that the measurement errors are modelled as White Gaussian zero-mean distributions. Whereby, to solve this problematic, the measurement vector is proposed to be modified as follows in 3-14:

$$
\begin{array}{r}
\tilde{z}^{\prime}{ }_{k}=\left(\tilde{\rho}_{k, G P S}^{1}-\mu(\widehat{M N P}), \ldots, \tilde{\rho}_{k, G P S}^{m}-\mu(\widehat{M N P}), \ldots, \tilde{\rho}_{k, G A L}^{N}\right. \\
\left.-\mu(\widehat{M N P}), \tilde{\dot{\rho}}_{k, G P S}^{1}, \ldots, \tilde{\dot{\rho}}_{k, G P S}^{m}, \ldots, \tilde{\dot{\rho}}_{k, G A L}^{N}\right)
\end{array}
$$

The new measurement vector is obtained modifying the pseudorange measurements removing the mean of the multipath and noise joint error distribution presented in Table 1, in order to obtain a zero-biased error distribution model. Then, the resulting distribution is over bounded by a zero-mean Gaussian distribution whose, as performed for LOS case.

The resulting equations for the pseudorange and pseudorange-rate measurement variances are described in 3-15 and 3-16, respectively.

$$
\begin{gathered}
\sigma_{\rho_{C}^{i}}^{\prime 2}[k]=\left(\sigma_{\overparen{M N_{u}^{i}}}^{2}[k]\right)+\sigma_{C, U R A^{i}}^{2}[k]+\sigma_{C, I^{i}}^{2}[k]+\sigma_{C, T^{i}}^{2}[k] \\
\sigma_{\dot{\rho}^{i}}^{\prime 2}[k]=\left(\sigma_{\widehat{M N}}^{2}[k]\right)
\end{gathered}
$$

where $\sigma_{\widehat{\mathrm{MNP}}}^{2}$ and $\sigma_{\widehat{\mathrm{MN}}}^{2}$ are the variance of the isolated pseudorange and pseudorange-rate Multipath plus AWGN noise error, from the proposed model in 2.2 .

The new Measurement Noise Covariance Matrix is thus defined as follows:

$$
R_{k}^{\prime}=\operatorname{diag}\left(\sigma_{C, \rho^{1}}^{\prime 2}[k], \ldots, \sigma_{C, \rho^{N}}^{\prime 2}[k], \sigma_{C, \dot{\rho}^{1}}^{\prime 2}[k], \ldots, \sigma_{C, \dot{\rho}^{N}}^{\prime 2}[k]\right)
$$

\subsection{Proposed EKF Model Results}

In this section, the PVT performances of the proposed basic EKF, using the theoretical $R$ matrix (3-11) and with the modified $R^{\prime}$ matrix (3-16), based on the realistic knowledge of MP and noise joint error distributions from pseudorange and pseudorange-rates are presented.

The PVT estimator is applied on a dataset collected in the Toulouse sub-urban and urban area, obtained as stated in section 2.2.1 [7]. The performances are evaluated with respect to the data collected by a Novatel \& SPAN receiver [7], which is used as a reference receiver in the Multipath and noise isolation methodology with an accuracy at the decimetre level. The Root Mean Square Error (RMSE) is used as a performance metric, as resumed in Table 3.

The proposed EKF algorithms are compared to:

- gLAB (GNSS-Lab Tool), EKF software (single-constellation, GPS);

- RTKlib Least Square (LS) software;

- ublox M8T real receiver (dual constellation, GPS+Galileo) position measurement collected during the data campaign. 
As summarized in Table 3, the proposed standard EKF has comparable performances with respect to the RTKlib solutions, while the gLAB has lower performances. The performances are worse with the respect of the real ublox receiver since the RMSE is $15 \mathrm{~m}$ and the ublox RMSE is around $6.7 \mathrm{~m}$.

Applying the proposed EKF with the modified $R^{\prime}$ matrix, it is noted an increase of performances compared to the standard EKF, around 30\%. Even if it is able to increase the performances of the PVT estimation, the resulting performances are not comparable with respect to the ublox performances, 10.4 and 6.7, respectively.

\begin{tabular}{|c|c|}
\hline Position Estimation & RMSE $[m]$ \\
\hline Ublox M8T & 6,735 \\
\hline gLAB & 31,194 \\
\hline RTKlib (LS Solution) & 12,094 \\
\hline Proposed Method (1) $R_{k}$ & 14,9744 \\
\hline Proposed Method (2) $R_{k}^{\prime}$ & $10,4852$ (Increased precision by $\sim 30 \%)$ \\
\hline
\end{tabular}

Table 3 - RMSE of the Proposed EKF with the basic Measurement Noise Error Covariance Matrix in orange, the Proposed EKF with the modified ) $R_{k}^{\prime}$ in green, compared to several PVT software and a real ublox M8T receiver.

\section{Satellite Selection}

The last section provides the Extended Kalman Filter state description, measurement vector description, and finally it provides also a possible modification of the Measurement Covariance Matrix based on the knowledges in 2.2. In the section 4.1, a review of the possible impairments impacting the precision of the Kalman Filter estimation is analysed, followed by the introduction of the Satellite Measurement Selection concept, which leads to the proposition of several methods provided to both try to improve the PVT accuracy with a low-complex method and to pose the basis of a possible systematic Satellite Selection method for low-cost GNSS receivers.

\subsection{Dependencies of the Kalman Filter estimation Precision}

The precision of the Kalman Filter estimations depends on two different factors:

- the precision of the state propagation model and,

- the precision of the measurement innovation model.

First, the precision of the state propagation model is necessary to better model the dynamics of the state under estimation; second, the precision of the measurement vector is important to reduce the possible impairments introduced by the a-priori state propagation based on the innovation thanks to the use of real measurements, reducing, therefore, the a-priori uncertainties. In fact, even with a well-designed model of the propagation states, the estimation uncertainties will grow in time if the a-priori estimations are not corrected by the measurements. However, the measurements precision degrades significantly in the urban environment, leading to a lack of PVT estimation accuracy. Focusing on the PVT performances of a low-cost receiver in the urban environment, it is fundamental to analyse how the error affect the measurements and to explore some innovative low-complex solutions to reduce the impact of the measurement errors.

The precision of the measurement innovation vector is based on two different criteria:

- The quality of the satellite measurement, which could be classified a-priori by the $C / N_{0}$ of the received signal, the elevation angle of the satellite, the NLOS/LOS received signal conditions. A satellite measurement featured by low $C / N_{0}$ and/or low elevation angle and/or NLOS received signal conditions, could be a-priori defined as "bad" satellite in the measurement vector. The presence of "bad" satellites leads to a certain level of estimates accuracy degradation. This problematic could be reduced by the realistic knowledges of the measurement errors in the given environment, as focused in the first part of the actual work.

- The geometry of the set of satellites used to make the innovation of the state. A poor signal-geometry, reduces the accuracy of the estimates. Usually, in the urban environments, the number of satellites in view 
is reduced and these satellites are mostly positioned to similar sky portion, reducing systematically the signal-geometry.

In the next section a resume of the method applied to reduce the impact of the "bad" satellites is presented. Successively, the satellite selection criteria, based on a trade-off between the selection of the satellite measurements and the knowledge of the satellite signal-geometry, is discussed.

\subsection{Satellite Selection: Motivations}

As discussed in section 3.5, the impact of the so-called "bad" satellites should be reduced to increase the accuracy of the PVT solution. A way to reduce their impact is based on the knowledges the Signal Processing Module of a low-cost stand-alone GNSS receiver has of the measurement errors. Basically, the Processing Module could select a weight for the measurements, which is calculated by means of the knowledges of the satellite measurement error variances through the use of the Measurement Noise Covariance Matrix, as showed in 3.4. On the contrary, it is not able to select/exclude satellite measurements exploiting any different criteria. Therefore, the PVT solution will be estimated by leans of all the possible satellite measurements, weighted with respect to the a-priori knowledges of the measurement error model.

An innovative solution could be afforded if the Processing module has additional knowledges of the measurement errors. It could be possible to select only the satellite measurements determined to be useful measurement in order to improve the PVT solutions. Supposing that the Signal Processing Module is built with a systematic methodology to characterize the LOS/NLOS signal conditions related to the satellite measurement. Then, a possible satellite selection criterion could be inspected: as an example, it is possible to apply a weight only on the satellite measurement classified as LOS and remove the satellite measurements classified as NLOS. The possibility to apply a systematic selection of only a portion of satellite measurements instead of taking the whole set of satellite measurements is the so-called Satellite Selection criteria.

The Satellite Selection could be also used to improve the PVT accuracy taking into account the trade-off between the exclusion of the "bad" satellites and the overall satellite-geometry factor: the basic satellite selection based on the LOS received signal conditions, depicted in section 4.4, could improve the PVT accuracy but has a significant drawback: as already stated, in the urban environment the number of satellites in the LOS visibility usually is reduced with the respect of open space environment. Sometimes only few satellites are in view, therefore the satellite measurement innovation vector is usually affected by poor geometry.

Removing a-priori satellite measurements considered NLOS could not be an optimal solution: a smaller set of satellite is going to be used to make the innovation, decreasing the quality of the signal geometry. The related PVT solution could be worst even if the estimator is excluding all the NLOS satellites.

A smart Satellite Selection method could be the way to select the satellite measurement in order to reduce the impact of the "bad" satellite measurements without impacting the quality of the satellite measurement geometry, which is however limited in the urban environments. In the section 4.1, several Satellite Selection Methods are proposed and analysed.

\subsection{Satellite Selection methods}

Following the final assumptions derived in section 4, this section presents some Satellite Selection Methods analysed in this work. The satellite selection methods are divided in two groups:

1. The Satellite Selection Methods based only on knowledges of the GNSS error models in the urban environment and/or a signal-geometry metric;

2. The Satellite Selection Methods based on the a-posteriori reference SPAN position in addition to the knowledges of the GNSS error models in the urban environment and/or a signal-geometry metric.

The first group contains:

- A low-complex method based on the LOS satellite measurement selection. As previously mentioned, the easiest way to exploit the knowledges on the measurement models and the NLOS/LOS received signal condition classification is removing all the satellite measurements defined to be NLOS. The method is presented in section 4.4 . 
- A complex systematic Satellite Selection based on the minimization of such a Geometrical Weight, which exploits also the knowledges of the realistic GNSS error model. Two different Geometrical Weight Metric are used, Weighted Position DOP and Kalman error covariance matrix, depicted in Section 4.5

The second group is used to make analysis, derive some possible insights, and put some basis of the potential improvements provided by the Satellite Selection, exploiting the reference receiver position, in section 4.6:

- First, a reverse engineering operation is performed to obtain information provided by the satellite measurement set which minimize the RMSE, in Section 4.6.1.1. The performance given by this method is unachievable but provides insights to develop a potential high performing satellite selection method.

- Second, in order to make more analysis on the selection of NLOS and LOS satellites, another method based on the mixed selection of LOS satellite measurements and a smart selection of NLOS satellite measurements is analysed (Section 4.6.2).

\subsection{LOS Satellite Selection (LOS S.S.)}

The first method, treated in this section, is called LOS satellite selection. Section 4.4.1 describes the method while section 4.4.2 shows the results.

\subsubsection{LOS S.S. Proposed Methodology}

As the name suggests, the method is based on the selection of the satellite measurement set constituted of LOS satellite signal condition only. For this purpose, a $C / N_{0}$ threshold is defined to differentiate between LOS and NLOS measurement conditions: every measurement above the threshold is selected by default. For this purpose, in the previous work [7], the $C / N_{0}$ threshold selected was $35 \mathrm{~dB}-\mathrm{Hz}$. Therefore, in this work the empirical threshold proposed in the previous work is applied. The goal of the method is to try to improve the accuracy of the estimation using a low-complex solution, in order to be easily applied to low-cost GNSS receivers.

\subsubsection{LOS S.S. Results}

Table 4 illustrates the RMSE obtained using LOS satellite selection compared with the performances of the proposed EKF without any satellite selection and the ublox M8T performances. The use of LOS satellite selection improves the PVT accuracy: a RMSE reduction from $10.48 \mathrm{~m}$ to $7.4 \mathrm{~m}$ is observed, which means an accuracy improvement of roughly $30 \%$. Furthermore, the performances of the proposed EKF with the application of LOS satellite selection approaches the receiver performances, even if the accuracy of the ublox receiver estimated position is still higher.

Figure 6 presents the performances in terms of RMSE error of three proposed methods: EKF with R matrix (Proposed Method (1), Section 3.3) - blue line, EKF with R' matrix (Proposed Method (2), Section 3.4) - red line, and EKF with $\mathrm{R}^{\prime}$ matrix and LOS satellite selection (section 4.4) - yellow line. A sensible increase of PVT accuracy could be noticed from the initial EKF to the one with LOS satellite selection, roughly $55 \%$ of increase of accuracy.

Figure 7 and Figure 8 show the histograms of the size of satellite measurement set selected and excluded by the method. Note that the mean of the satellite measurement selected' size is around 10.

\begin{tabular}{|c|c|}
\hline Solution & RMSE $[\boldsymbol{m}]$ \\
\hline Ublox M8T & 6,735 \\
\hline Proposed Method (2) $\boldsymbol{R}_{\boldsymbol{k}}^{\prime}$ & 10,4852 \\
\hline LOS SS & 7,40 \\
\hline
\end{tabular}

Table 4 - RMSE error of the PVT solutions based on LOS Satellite Selection. 




Figure 6 - Plot of the RMSE error of PVT solution based on the Proposed Method (1), blue curve, the

Proposed Method (2), red curve, LOS satellite selection, yellow line

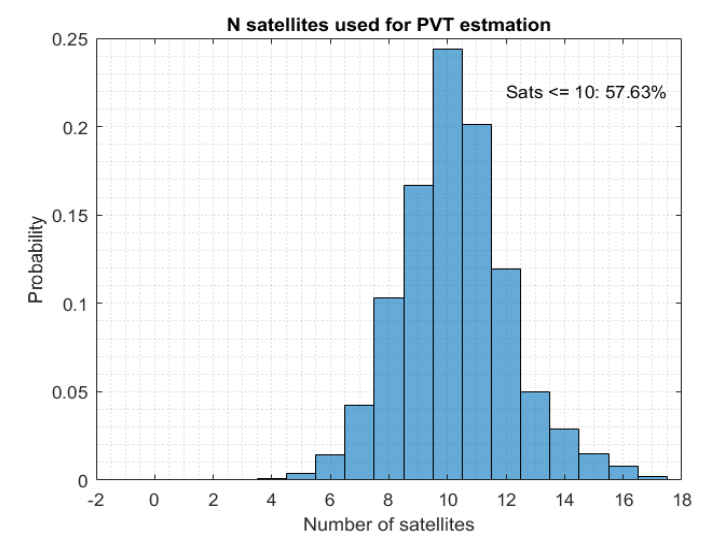

Figure 7 - Histogram of the selected satellite set size, used to provide the PVT estimation

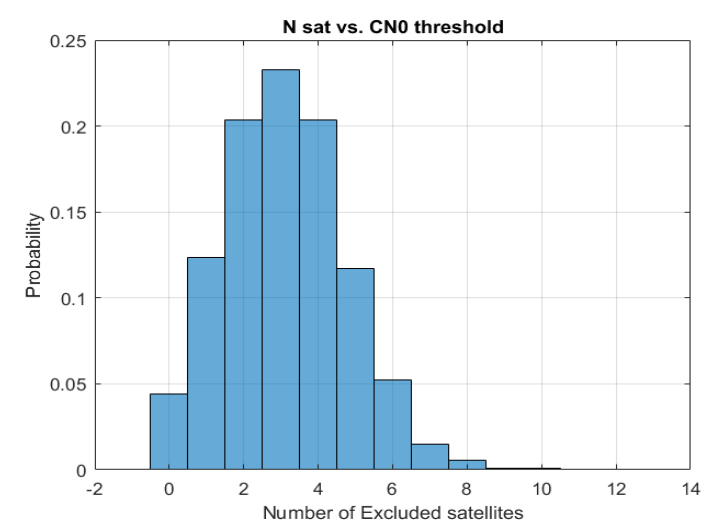

Figure 8 - Histogram of the excluded satellite set size

The LOS satellite selection improves the PVT estimation precision, has low-complexity and could be used in lowcost receivers. The most important upgrade needed by this method is a realistic and more reliable classification of LOS/NLOS received satellite conditions. Increasing these knowledges is vital to have a higher refinement of the PVT performances.

\subsection{Minimization of Weighted Geometrical Metric Satellite Selection (WGM S.S.)}

The LOS satellite selection, even if able to increase the performances of the PVT estimator, should be not able to select in a-priori way the best set of satellite measurements needed by the Processing module to get the best PVT performances. In fact, as already stated, the NLOS satellite measurement removal could be detrimental in the hypothesis of urban canyon, where the original set of measurement could be small, which also means a small set of LOS satellite measurements. Therefore, a method able to select the a-priori best set of satellite measurements from the combined knowledge of the geometry of the satellite measurements set and the realistic knowledges about the GNSS measurement error in urban environment, (quality of satellite measurement), should be inspected. The actual section inspects a method based on the minimization of two different Weighted Geometrical Metric. Section 4.5.1 presents the steps of the algorithm, then, the section 4.5.2 analysis the results.

\subsubsection{WGM S.S.: Proposed Methodology}

The goal of the method is to try to improve the accuracy of the PVT compared to the LOS satellite selection method by exploiting the minimization of a given Weighted Geometrical Metric. As a trade-off, an increase of the total satellite selection method complexity is allowed.

In the state of the art, for the Least Square algorithm, to have a-priori information on the quality of the measurement vector, a weighted geometrical metric is used which combines the knowledge of the Dilution of Precision (DOP), [1], [11] and the knowledge of the Measurement Noise Covariance Matrix, $R$, [1]. The resultant Metric is called 
Weighted DOP (WDOP) [12], [13]. Moreover, the Kalman Filter keeps track of the geometrical metric and the satellite measurement weights in a more complex way, balancing also the knowledges about the state propagation model, through the Innovated Kalman Error Covariance State Matrix [1].

The basic idea behind the satellite selection method analysed in this section is to try to select the set of satellites which minimize a Weighted Geometrical Metric, identified in the minimization of the Weighted Position DOP (WPDOP) or in the minimization of the Kalman Error Covariance Position State Matrix (Kalman $P^{+}$).

The minimization of Weighted Geometrical Metric is based on the sequent steps:

1. At a given epoch $k$, during the EKF innovation phase, the Processing Module selects all the possible combination of satellite measurement sets which fulfil the following conditions: the minimum satellite set size is equal to 5 satellites, while the maximum size goes from a minimum of 5 up to all the possible visible satellites.

2. The state vector error covariance matrix update applying the different satellite measurement set, is given by:

where

$$
P_{k}^{+{ }^{s e t}}=\left(I-K_{k}^{\text {set }_{i}} H_{k}^{\text {set }_{i}}\right) P_{k}^{-}
$$

- $\quad I$ is the identity matrix.

- $K_{k}^{\text {set }_{i}}$ is the Kalman gain defined using the selected satellite measurement set, at epoch $k$;

- $H_{k}^{\text {set }_{i}}$ is the observation matrix defined using the selected satellite measurement set, at epoch $k$;

- $\quad P_{k}^{-}$is the a-priori State Error Covariance Matrix.

3. Each satellite measurement set, $\operatorname{set}_{i}[k]$, selected by the Processing Module is used by the EKF to generate the innovation measurement vector, $\tilde{z}_{k}$, and is used to calculate the $\operatorname{WPDOP}_{\boldsymbol{k}}^{\text {set }_{i}}$, or the Kalman $P_{k}^{+}$, using the specific set of satellites.

$$
\begin{gathered}
\mathrm{WPDOP}_{k}^{s e t_{i}}=\sqrt{\operatorname{tr}\left[\left(H_{k}^{\text {set }_{i}^{T}} R_{k}^{{ }^{\text {set }_{i}}{ }^{-1}} H_{k}^{\text {set }_{i}}\right)^{-1}\right]_{3}} \\
P_{k}^{+ \text {set }_{i}}=\sqrt{\operatorname{tr}\left[P_{k}{ }^{+ \text {set }_{i}}\right]_{3}}
\end{gathered}
$$

where

- $H_{k}^{\text {set }}{ }_{i}$ is the Measurement Design Matrix using only the selected set of satellites, $\operatorname{set}_{i}[k]$, at a given epoch $k$;

- $R_{k}^{\prime s e t}$ is the Measurement Noise Covariance Matrix proposed in this work, (section 3.4), Matrix using only the selected set of satellites, $\operatorname{set}_{i}[k]$, at a given epoch $k$;

- $[\ldots]^{T}$ is the transpose operation;

- $[\ldots]^{-1}$ is the inverse operation;

- $\operatorname{tr}[\ldots]_{3}$ is the trace operation, limited only to the first three component of the matrix, which are linked to the position DOP and the position State Error Covariance Matrix

4. Among all the possible combinations of satellite sets, the one which minimize the WPDOP, $\operatorname{set}_{W P D O P \min }[k]$, or the Kalman $P_{p_{k}}^{+}$, $\operatorname{set}_{P^{+} \text {min }}[k]$ is chosen:

$$
\begin{aligned}
\operatorname{set}_{W P D O P \min }[k] & =\operatorname{set}_{i}\left[\min \left(\mathrm{WPDOP}_{k}^{\text {set }_{i}}\right)\right] \\
\operatorname{set}_{P^{+} \min }[k] & =\operatorname{set}_{i}\left[\min \left(P_{p_{k}}^{\text {set }_{i}}\right)\right]
\end{aligned}
$$

5. Finally, the resultant satellite set which minimize the given metric is used to finalize the EKF innovation phase:

$$
\begin{gathered}
s_{k+1_{W P D O P \min }}=E K F\left(\operatorname{set}_{W P D O P \min }\right) \\
s_{P^{+} \min }=E K F\left(\operatorname{set}_{P^{+} \text {min }}\right)
\end{gathered}
$$

The step 1 can be modified to establish a more elaborated rules that need to be fulfilled by a satellite set to be retained as candidate for the next steps. Therefore, the evaluated satellite measurement set could be constrained in several ways:

- The satellite measurement set size could be modified in order to contain a defined number of satellites; in the section 4.5.2 the satellite measurement size will be defined by the all visible satellites, only 5 and only 7 satellites.

- The satellite measurement set could be defined including a satellite measurement defined by different signal LOS/NLOS conditions. Section 4.5.3 in particular, analyses the performances of PVT estimation 
using the WPDOP minimization applied to a particular set of satellites obtained combining all the possible satellite measurements considered in LOS conditions and a random number of satellite measurements considered in NLOS conditions: all LOS satellite measurements plus one NLOS, two NLOS, and three NLOS satellite measurement.

\subsubsection{WGM S.S. Results: Different maximum satellite measurement size}

As stated in 4.5.1, the evaluation of the results is obtained applying:

1. the WPDOP minimization fixing the minimum size of the satellite measurement set to 5 satellites and the maximum size to:

1. all visible satellites;

2. 7 satellites;

3. 5 satellites.

2. the Kalman Error Covariance Position State Matrix minimization fixing the minimum size of the satellite measurement set to 5 satellites and the maximum size to all visible satellites.

Table 5 shows the related RMSE obtained as explained in point 1 and 2 of this section, compared to:

- RMSE of PVT estimation using LOS satellite selection.

- The performances of the proposed EKF without any satellite selection and.

- The ublox M8T performances.

The use of the WPDOP\Kalman Error Covariance Matrix minimization does not improve the precision of the PVT Estimations for any satellite set size. As stated in literature [13], the WPDOP is usually decreasing with respect to the number of satellites used to make the solution. Even if it is applied a weight which corresponds to a realistic knowledge of the GNSS measurement errors in the urban environment, this statement is still verified: a higher number of satellites corresponds to a lower WPDOP. Therefore, due to the WPDOP monotonic behaviour with respect to the number of satellites, to use the chosen metric as a standalone method is not a good solution.

In the same way, the minimization of the Kalman Error Covariance Position State Matrix, which acts similarly to the WPDOP minimization does not improve the precision of the PVT Estimations. From the previous observations, it could be stated that the Kalman filter makes a de-weighting of the "bad" measurement (in the case under exam with the respect of the received signal $C / N_{0}$ ), but it could not be able to find the right satellite in order to minimize the RMSE. In fact, comparing the results with the LOS method, it can be stated that the keeping all the satellites and just weighting them (de-weighting a-priori bad satellites, NLOS, with respect to good satellites, LOS) provides worse position performance than just removing the NLOS satellites.

Figure 9 presents the performance in terms of RMSE error of satellite selection based on the Kalman $P_{p_{k}}^{+}$ minimization and the performances of the LOS satellite selection: the blue curve is the RMSE of the proposed EKF with the application of the realistic knowledges about the GNSS error in the urban environment and the application of the LOS satellite selection; while the red curve is the same but with minimization of Kalman $P_{p_{k}}^{+}$ satellite selection. The LOS satellite selection has higher performances in comparison with the second one.

Figure 10 presents the performances in terms of RMSE error of satellite selection based on the WPDOP minimization: the blue curve is the RMSE of the proposed EKF with the application of the WPDOP minimization satellite selection with the maximum size of satellite set equal to all visible satellites; the red curve is the same but the maximum size of satellite set equal to 5, and for the yellow curve the maximum size of satellite set equal to 7 . It is impossible to notice any improvements between the different cases. 


\begin{tabular}{|c|c|}
\hline Solution & RMSE $[\mathrm{m}]$ \\
\hline Ublox M8T & 6,735 \\
\hline Proposed Method (2) $R_{k}^{\prime}$ & 10,4852 \\
\hline LOS selection & 7,40 \\
\hline Min WPDOP all sat & 10,91 \\
\hline Min WPDOP < =7 & 10 \\
\hline Min WPDOP = 5 & 10,43 \\
\hline Min P Kalman & 10,9 \\
\hline
\end{tabular}

Table 5 - RMSE of WGM SS, with different maximum satellite set size compared to the Proposed Method (2), the LOS SS and the ublox receiver

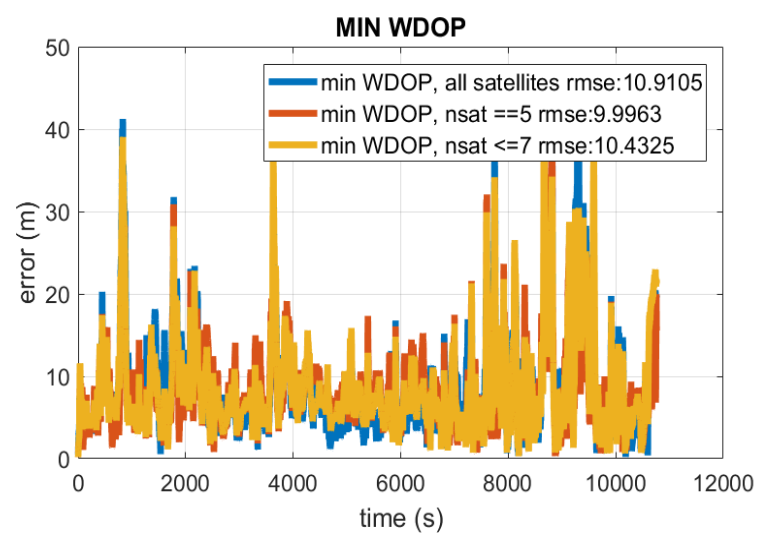

Figure 9 - RMSE of WPDOP SS using all visible satellites, blue curve, only a set of 5 satellites, red curve, a set of 7 satellites, yellow curve



Figure 10 - RMSE of KP SS using all visible satellites, blue curve, and LOS SS, red curve

\subsubsection{WGM S.S. Results: Different combination of LOS and NLOS satellite measurement}

The section presents the evaluation of the results obtained applying the WPDOP minimization applied to particular set of satellites obtained combining all the possible satellite measurements considered in LOS conditions and a random number of satellite measurements considered in NLOS conditions: all LOS satellite measurements plus one, two and three NLOS satellite measurement.

Table 6 shows the related RMSE obtained as explained above compared with PVT performances of:

- WPDOP minimization satellite selection using all the visible satellites,

- LOS satellite selection,

- $\quad$ proposed EKF without any satellite selection

- ublox M8T performances

Reducing the number of NLOS satellite measurements improves the performances of the PVT estimator; using all the LOS satellite measurement and just one NLOS satellite measurement, when possible, has RMSE error around $8.84 \mathrm{~m}$, with an increase of performances around $15 \%$ with respect to the Proposed method without any satellite selection and an increase of performance around 18\% compared to the WPDOP minimization using all visible satellites. Conversely, the LOS satellite selection has in any case higher performances compared to the combinations proposed. The performances degrade if the satellite set contains a higher number of NLOS satellite measurement. As stated in the previous section, the NLOS satellites measurements used in the Kalman Filter, even de-weighted, have a relevance in the PVT estimation when used together with all the LOS satellites.

Figure 11 presents the performance in terms of RMSE of the satellite selection based on the WPDOP minimization with a base satellite measurement set formed by all LOS satellites and a given number of NLOS satellites: the blue 
curve is the RMSE of the proposed EKF with the application of the WPDOP minimization satellite selection with the maximum size of satellite set equal to all LOS satellites; the red curve is the same but the maximum size of satellite set equal to all LOS satellites and one NLOS satellites, same for the yellow curve but two NLOS satellites and finally three NLOS satellites in the case of magenta line.

\begin{tabular}{|c|c|}
\hline Solution & RMSE $[\mathrm{m}]$ \\
\hline Ublox M8T & 6,735 \\
\hline Proposed Method (2) $R_{k}^{\prime}$ & 10,4852 \\
\hline LOS selection & 7,40 \\
\hline ALL VISIBLE SAT & 10,91 \\
\hline ALL LOS + 1 NLOS & 8,84 \\
\hline ALL LOS + 2 NLOS & 9,7 \\
\hline ALL LOS + 3 NLOS & 10,52 \\
\hline
\end{tabular}

Table 6 - RMSE of WGM SS, with different maximum satellite set defined by: all LOS plus 1 NLOS, 2 NLOS and 3 NLOS



Figure 11 - RMSE of WPDOP SS, with different maximum satellite set defined by: all LOS, blue curve, all LOS plus 1 NLOS, red curve, 2 NLOS, yellow curve and 3 NLOS, magenta curve.

\subsection{Additional Analysis: Satellite Selection based on Minimization of RMSE}

The study of the satellite selection methods described until now shows that the LOS satellite selection improves the accuracy of the PVT estimator but it is not able to reduce the RMSE error to the 1m-level. An a-priori satellite selection based on the WPDOP minimization and Kalman Error State Covariance Matrix does not improve the PVT accuracy, therefore it is needed to add further information regarding the quality of satellite measurements, otherwise the minimization of the Weighted Geometrical Matrix is unproductive.

The NLOS satellite measurements, even de-weighted by the EKF thanks to the realistic model proposed in this work, are still impacting the PVT estimation performances. A gradual removal of NLOS satellite measurements coincides with an increase of performances. Based on the previous observations, the PVT performance obtained using LOS satellite selection could not be overtaken by others low-complex methods. It is necessary to research an innovative way to exploit the Satellite Selection criteria in order to improve systematically the PVT accuracy.

To do so, a possible initial way to research for additional information and to build the foundation of an optimal satellite selection set method could be the application of a reverse engineering analysis. The reverse analysis is based on the satellite measurements selection which minimizes at each epoch the RMSE. Moreover, if a systematic way to exploit a trend in the observed results could be proved in the future works, having knowledges about the best satellite sets given the minimum RMSE it should be also possible to provide an analysis of the PVT performances modifying the evaluated satellite measurement set, combining the LOS satellite measurements with the NLOS satellite measurements which minimize the RMSE.

In section 4.6.1 minimization of the RMSE Satellite Selection is presented. Section 4.6.1.1 presents the insights and the comments related to this approach. While, the selection of LOS and smart selected NLOS is discussed in 4.6.2. Finally, the results of the last approach are analysed in 4.6.2.1.

\subsubsection{Minimum RMSE Satellite Selection: Proposed Methodology}

In this section, the minimization of the RMSE Satellite Selection algorithm is presented. Basically, the proposed method calculates all the possible satellite set combinations to calculate the position estimation. Hence, knowing the reference SPAN position estimation, it is selecting the set of satellite measurements which minimizes the RMSE. 
The minimization of the RMSE Satellite Selection is based on the sequent steps:

- At a given epoch $k$, during the EKF innovation phase, the Processing Module selects all the possible combination of satellite measurement sets, set $_{i}[k]$, which fulfil the following conditions: the minimum satellite set size is equal to 5 satellites, while the maximum size is equal to all the possible visible satellites.

- $\quad$ Each satellite measurement set, set $t_{i}[k]$, selected by the Processing Module is used by the EKF to generate the innovation measurement vector, $\tilde{z}_{k}$ :

$$
p_{k+1_{\text {set }_{i}}}=\operatorname{EKF}\left(\operatorname{set}_{i}[k]\right)
$$

- Hence, knowing the reference SPAN position estimation, $p_{k+1}$ SPAN , the calculation of the RMSE using $p_{k+1}$ SPAN as a reference solution is performed, $R M S E_{k+1}^{s e t_{i}}$.

- The minimum RMSE satellite measurement set, $\operatorname{set}_{\operatorname{minRMSE}}[k]$, is selected by minimizing $R M S E_{k+1}$ set $_{i}$.

$$
\operatorname{set}_{\operatorname{minRMSE}}[k]=\min \left[R M S E_{k+1}^{\text {set }_{i}}\right]
$$

\subsubsection{RMSE Minimization Results}

The section evaluates the results obtained applying the RMSE minimization satellite selection.

Table 7 presents the RMSE obtained using RMSE minimization (Figure 12), compared with the performances of the proposed EKF without any satellite selection and the ublox M8T performances. As shown in the table, theoretically, and unachievable with a systematic satellite selection method, the minimum RMSE is around $0.4 \mathrm{~m}$. The obtained results, if systematically proved by a trend, could provide some new information to be exploited in a feasible satellite selection method to increase the PVT accuracy for a stand-alone GNSS receiver.

It is important to remark that the application of this method should be verified, as explained before, by the possibility to extract systematically a possible trend in the satellite measurement set. Next work will be focused on that, if possible. If it could be supposed an hypothetic systematic trend, some consideration could be extracted from the analysis of the results. More than being focused on the potential increase of performances, the study could tell also several information about the best satellite measurements set using to get the minimum RMSE.

Figure 13 and Figure 14 show the histograms of the size of LOS+NLOS and NLOS satellite measurement sets selected by the method, respectively. The minimum RMSE is usually reached using a restricted set of satellites, more than half of time only 5 satellite measurements, instead of using a larger set of satellites. In these cases, the measurement redundancy is avoided. Moreover, a restricted number of satellite measurements considered in NLOS conditions are used among the LOS satellite measurements (in average 1.2 NLOS measurements) to obtain the minimum RMSE. This means that the NLOS should not be excluded a-priori, but a satellite selection method which is able to select the NLOS satellite measurement, (usually considered "bad" satellites defined by low weight), is needed. It remains to be seen, if a systematic method choosing as often as possible the required satellite set can be developed.

\begin{tabular}{|c|c|}
\hline Solution & RMSE $[\boldsymbol{m}]$ \\
\hline Ublox M8T & 6,735 \\
\hline Proposed Method (2) $\boldsymbol{R}_{\boldsymbol{k}}^{\prime}$ & 10,4852 \\
\hline Min RMSE & 0,37021 \\
\hline
\end{tabular}

Table 7 - Minimum RMSE compared to the Proposed Method (2) and the ublox receiver RMSE

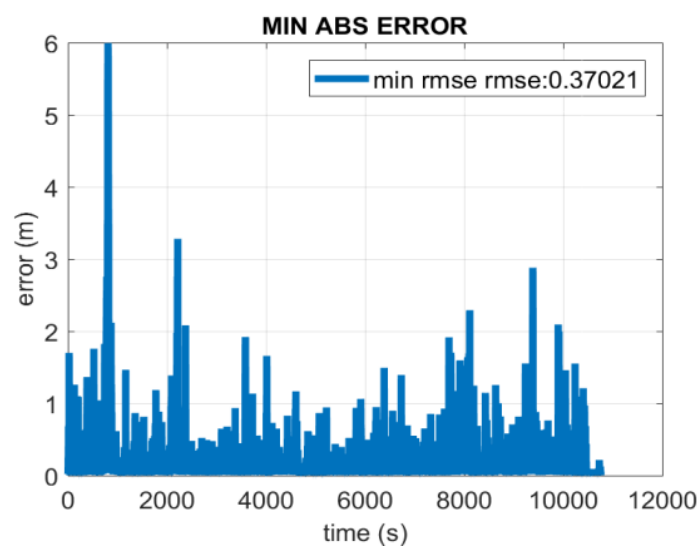

Figure 12 - Minimum RMSE 


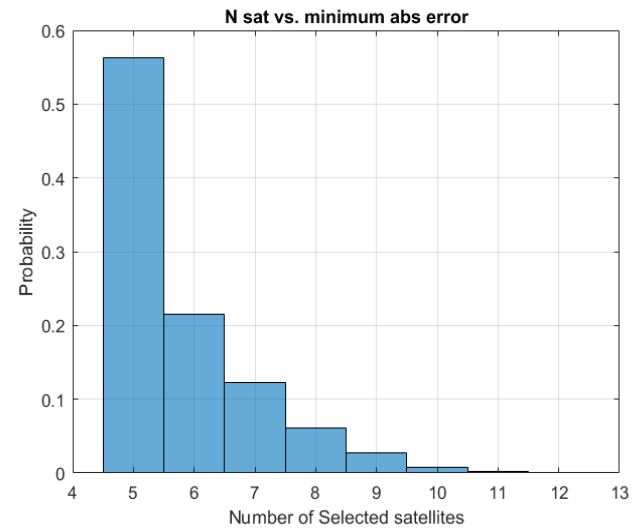

Figure 13 - Histogram of the selected satellite set size, used to provide the PVT estimation which minimizes the RMSE

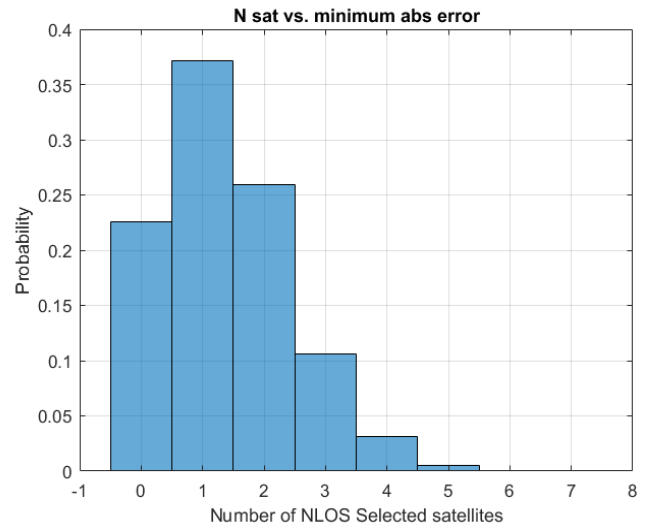

Figure 14 - Histogram of the NLOS selected satellite set size, used to provide the PVT estimation which minimizes the RMSE

\subsubsection{Smart LOS and NLOS satellite selection: Proposed Methodology}

As stated in the section 4.6.1.1, it should be required a satellite selection method which is able to select the NLOS satellite measurements useful to increase to PVT performances. An example of NLOS satellite selection is provided by the proposed method in section 4.5.3, where the NLOS satellite measurement are selected minimizing the WPDOP metric. As already stated, the method does not introduce any improvement.

As also provided in the section 4.6.1.1, only a restricted set of LOS satellite should be used to make the PVT estimation. It is then mandatory analyse also the impact of LOS satellite measurements on the PVT accuracy, because the selection of LOS satellite instead of taking all the possible LOS satellite measurement increases the complexity of a possible feasible method.

A possible way to study the impact of LOS and NLOS satellite measurements on the PVT performances could be trying to define the satellite set by using all the possible LOS satellite measurements followed by a smart NLOS satellite selection. In this specific case, the selected NLOS satellite measurements are the satellite measurements taken in 4.6.1.1 to minimize the RMSE.

\subsubsection{Mixed LOS and smart NLOS S.S. Results}

The section is devoted to the evaluation of the results obtained applying the LOS satellite selection and a smart NLOS satellite selection based on the NLOS measurements which minimize the RMSE error. Table 8 shows the RMSE obtained using the proposed method, compared with the performances of the proposed EKF without any satellite selection and the ublox M8T performances. Figure 15 shows the RMSE evaluation in time of PVT solution using the proposed method.

Several comments could be afforded regarding the analysis under exam:

- The PVT performances applying the proposed method are comparable with the ublox M8T receiver performances, better than LOS satellite selection, $6.6 \mathrm{~m}$ versus $7.4 \mathrm{~m}$.

- The use of smart selected NLOS satellite measurements has a positive impact on the PVT estimation accuracy. Assuming that there is a way to select these optimal NLOS measurements, the WDOP is not a good method to select NLOS satellite measurements;

- Using all the possible LOS satellite measurements instead of a smart selected set of LOS measurements impair the possibility to reach high-level of PVT accuracy. It is then needed a methodology to refine the characterization of LOS satellite measurements in order to choose them in a smarter way since in addition to select the correct NLOS we must remove the LOS which hide the impact of the NLOS.

- The use of all LOS satellites in the Kalman Filter may mask the NLOS satellite which are optimal to calculate the PVT solution: NLOS satellites are de-weighted w.r.t. to the better satellites due to the "apriori" worst sigma, even if some NLOS are better than some LOS for the PVT computation.

- It is finally mandatory find a new classification of "useful" satellite measurement based on more information in order to study a systematic and effective satellite selection algorithm. 


\begin{tabular}{|c|c|}
\hline Solution & RMSE $[\boldsymbol{m}]$ \\
\hline Ublox M8T & 6,735 \\
\hline Proposed Method (2) $\boldsymbol{R}_{\boldsymbol{k}}^{\prime}$ & 10,4852 \\
\hline LOS sat + NLOS min rmse & 6,6 \\
\hline
\end{tabular}

Table 8 - RMSE obtained using all LOS satellites and the NLOS set of satellites compared to the Proposed Method (2) and the ublox receiver RMSE



Figure 15 - RMSE of WPDOP SS using all visible satellites, blue curve versus RMSE obtained using all LOS and a NLOS set of satellites

\section{Conclusions and Future works}

The first part of this work is based on the application of Multipath and noise joint error isolation methodology to obtain the isolation and, afterward, the characterization of the Doppler frequency measurements. The characterization is based on the evaluation of the empirical probability density function characterized by several received signal $C / N_{0}$ bands.

The resulting distributions are characterized by a Gaussian and zero mean behaviour.

Second, a low-cost EKF GNSS algorithm is implemented, exploiting the knowledges of the a priori multipath error component plus noise joint error distributions of the pseudorange and pseudorange-rate measurements; by means of the Noise Measurement Covariance Matrix modification. The resulting modified EKF model provides an increase of precision of $\sim 30 \%$ compared to the same EKF model based on the theoretical Measurement Noise Covariance Matrix.

Moreover, some satellite selection methods are presented and analysed in this work. The first is a low-complex method and is based on the LOS satellite measurement selection. The LOS satellite selection improves the PVT estimation precision by almost $30 \%$, has low-complexity and could be used in low-cost receivers. The second method is a more-complex systematic satellite selection based on the minimization of a Weighted Geometrical Metric, Weighted Position DOP and the Kalman Error Covariance Position State Matrix. The use of the WPDOP\Kalman Error Covariance Matrix minimization does not improve the precision of the PVT Estimations. As analysed from this method, it seems that it is better to remove satellite measurements than keeping them all, applying only the standard EKF de-weight approach on the "bad" satellite measurements.

The second section is used to make some analysis of the possible satellite selection performance potential, exploiting the knowledges of the reference receiver position: the goal of the study is to get some insight or hints from the proposed approach in order to see a possible trend that allows to determine a potential systematic satellite selection method. The proposed approach is based on:

- Firstly, a reverse engineering operation, performed to assess the ideal maximum performances of the satellite selection: the method selects the set of satellites giving the minimum position RMSE. The obtained insights reveal, if a trend could be systematically observed and derived, a potential powerful method to increase the PVT accuracy for a stand-alone GNSS receiver. The minimum RMSE is usually reached using a restricted set of satellites, instead of using a larger set of satellites. Moreover, a restricted number of satellite measurements considered in NLOS conditions are used among the LOS satellite measurements.

- Secondly, in comparison with the method proposed in the first group, a method based on the selection of LOS satellite measurements and a smart selection of NLOS satellite measurements is analysed. The 
selected NLOS satellites measurements are the NLOS measurement which potentially minimize the RMSE per each time epoch. The PVT performances applying the proposed method are comparable with the ublox M8T receiver performances, better than LOS satellite selection. The use of smart selected NLOS satellite measurements has a positive impact on the PVT estimation accuracy. Moreover, the good influence of the NLOS satellite measurements should probably be masked by some of the LOS satellite measurements of the EKF. Therefore, the conclusion is that if a potential method could be derived from these preliminary studies, it should also remove the LOS satellite measurements to allow the EKF to exploit the useful NLOS satellite measurements. It is finally mandatory find a new classification of "useful" satellite measurement based on more information in order to study a systematic and effective satellite selection algorithm.

The future works will focus on the improvement of the realistic knowledge regarding the Pseudorange and Doppler frequency error measurements in urban environment increasing the dataset with new data campaigns, with enhanced characteristics based on larger higher sample frequency of the measurements and a more accurate LOS/NLOS receiver signal condition detection. At the same time, also the PVT estimator should be improved by means characterizing the multipath errors from possible correlation in time and space. Finally, a more complex PVT solution will be implemented taking into account the non-Gaussianity of the pseudorange NLOS multipath error measurements, as the exploitation of the Particle Filter. 


\section{BIBLIOGRAPHY}

[1] P. D. Groves, "Principles of GNSS, inertial, and multisensor integrated navigation systems, 2nd edition," IEEE Aerosp. Electron. Syst. Mag., vol. 30, no. 2, pp. 26-27, 2015.

[2] S. Tay, J. Marais, "Weighting models for GPS Pseudorange observations for land transportation in urban canyons To cite this version : HAL Id : hal-00942180 Weighting models for GPS Pseudorange observations for land transportation in urban canyons," 2014.

[3] N. Zhu, D. Betaille, J. Marais, and M. Berbineau, "Extended Kalman Filter (EKF) Innovation-Based Integrity Monitoring Scheme with C/N 0 Weighting," IEEE 4th Int. Forum Res. Technol. Soc. Ind. RTSI 2018 - Proc., pp. 1-6, 2018.

[4] A. Pirsiavash, A. Broumandan, G. Lachapelle, and K. OrKeefe, "Detection and De-weighting of Multipath-affected Measurements in a GPS/Galileo Combined Solution," pp. 1-11, 2019.

[5] P. D. Groves and Z. Jiang, "Height Aiding, C / N 0 Weighting and Consistency Checking for GNSS NLOS and Multipath Mitigation in Urban Areas ," J. Navig., vol. 66, no. 5, pp. 653-669, 2013.

[6] C. Cai and Y. Gao, "A Combined GPS/GLONASS Navigation Algorithm for use with Limited Satellite Visibility,” J. Navig., vol. 62, no. 4, pp. 671-685, 2009.

[7] E. R. Matera, A. Garcia-Pena, O. Julien, C. Milner, and B. Ekambi, "Characterization of line-of-sight and non-line-of-sight pseudorange multipath errors in urban environment for GPS and galileo," ION 2019 Int. Tech. Meet. Proc., pp. 177-196, 2019.

[8] T. Singhal, A. Harit, and D. Vishwakarma, "Kalman filter implementation on an accelerometer sensor data for three state estimation of a dynamic system," Int. J. Res. Eng. Technol., vol. 1, no. 6, pp. 330334, 2012.

[9] C. Gioia and D. Borio, "A statistical characterization of the Galileo-to-GPS inter-system bias," J. Geod., vol. 90, no. 11, pp. 1279-1291, 2016.

[10] Enik Shytermeja, "Design and Performance of a GNSS Single-frequency Multi-constellation Vector Tracking Architecture for Urban Environments," 2017.

[11] S. K. Biswas, L. Qiao, and A. G. Dempster, "Effect of PDOP on performance of Kalman Filters for GNSS-based space vehicle position estimation," GPS Solut., vol. 21, no. 3, pp. 1379-1387, 2017.

[12] C. S. Chen, Y. J. Chiu, C. T. Lee, and J. M. Lin, "Calculation of weighted geometric dilution of precision," J. Appl. Math., vol. 2013, 2013.

[13] H. Sairo, D. Akopian, and J. Takala, "Weighted dilution of precision as quality measure in satellite positioning," vol. 150 , no. $6,2003$. 